\title{
Validation of Enthalpy-Entropy Compensation Mechanism in Partial Amide Bond Rotation
}

\author{
Jacob Guerraㄹ, Bhvandip Bajwa ${ }^{1}$, Prarthana Kumar ${ }^{1}$, Salvador Vazquez ${ }^{1}$, \\ Viswanathan.V. Krishnan ${ }^{1,2, *}$, and Santanu Maitra, ${ }^{1, *}$ \\ ${ }^{1}$ Department of Chemistry, California State University, Fresno CA 93740; \\ ${ }^{2}$ Department of Pathology \& Laboratory Medicine, University of California \\ Davis, Davis CA 95616
}

\section{Supporting Information}

Table S1: Experimental data - Temperature vs. $k_{\text {ex }}$.

Table S2: Estimated activation enthalpy and entropy of all the molecules.

Figure S1: EEC plot for the sub-groups

Figure S2: NMR $\left({ }^{1} \mathrm{H}\right.$ and $\left.{ }^{13} \mathrm{C}\right)$ spectra of all the molecules.

${ }^{*}$ Correspondence to V.V. Krishnan (krish@csufresno.edu) or S. Maitra (smaitra@csufresno.edu) 
Table S1: List of $k_{\mathrm{ex}}\left(\mathrm{s}^{-1}\right)$ values as a function of temperature $(\mathrm{K})$

\begin{tabular}{|l|c|c|c|c|c|c|c|c|c|c|c|}
\hline Temperature (K) & 277.50 & 282.89 & 288.27 & 293.65 & 299.03 & 304.42 & 309.80 & 315.18 & 320.56 & 325.95 & 331.33 \\
\hline
\end{tabular}

\begin{tabular}{|l|c|c|c|c|c|c|c|c|c|c|c|}
\hline $\mathrm{m}: \mathrm{NH} 2: \mathrm{B}$ & 2 & 3 & 5 & 10 & 15 & 29 & 39 & 55 & 73 & 85 & 108 \\
\hline $\mathrm{m}: \mathrm{OCH} \cdot \mathrm{B}$ & 331 & 472 & 619 & 780 & 975 & 1285 & 1524 & 1802 & 2100 & 2500 & 3000 \\
\hline
\end{tabular}

$\mathrm{m}: \mathrm{CH} 3: \mathrm{B}$

$m: C F 3: B$

$\mathrm{m}: \mathrm{NO} 2 \mathrm{~B}$

$\mathrm{H}: \mathrm{B}$

$\mathrm{O}: \mathrm{NH} 2: \mathrm{B}$

$\mathrm{O}: \mathrm{OCH} 3: \mathrm{B}$

$\mathrm{O}: \mathrm{CH} 3: \mathrm{B}$

$\mathrm{O}: \mathrm{F}: \mathrm{B}$

$\mathrm{O}: \mathrm{CF} 3: \mathrm{B}$

$\mathrm{m}: \mathrm{NH2}: \mathrm{C}$

(OHB:C

$\mathrm{m}: \mathrm{F}: \mathrm{C}$

m:CF3:C

$\mathrm{m}: \mathrm{NO} 2 \cdot \mathrm{C}$

$\mathrm{H}: \mathrm{C}$

$\mathrm{O}: \mathrm{NH} 2: \mathrm{C}$

$\mathrm{O}: \mathrm{OCH} 3: \mathrm{C}$

$\mathrm{O}: \mathrm{CH} 3: \mathrm{C}$

$\mathrm{o:F:C}$

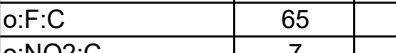

(n:

$\mathrm{m}: \mathrm{OCH} 3: \mathrm{D}$

$\mathrm{m} \cdot \mathrm{CH} 3 \cdot \mathrm{D}$

$m: F: D$

\begin{tabular}{|l|l|l|l|l|l|l|l|l|l|}
\hline 546 & 640 & 788 & 971 & 1125 & 1400 & 1627 & 1900 & 2031 & 2215 \\
\hline
\end{tabular}

\begin{tabular}{|l|l|l|l|l|l|l|l|l|l|l|}
425 & 546 & 640 & 788 & 971 & 1125 & 1400 & 1627 & 1900 & 2031 & 2215 \\
\hline 178 & 260 & 374 & 491 & 675 & 900 & 1191 & 1450 & 1750 & 2200 & 2700 \\
\hline
\end{tabular}

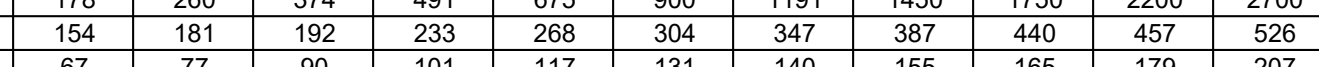

\begin{tabular}{|c|c|c|c|c|c|c|c|c|c|c|}
\hline 67 & 77 & 90 & 101 & 117 & 131 & 140 & 155 & 165 & 179 & 207 \\
\hline 2.8 & 7.1 & 13.7 & 19.5 & 32 & 75 & 123 & 201 & 282 & 417 & 590 \\
\hline
\end{tabular}

\begin{tabular}{|c|c|c|c|c|c|c|c|c|c|c|}
\hline 90 & 139 & 188 & 206 & 356 & 477 & 658 & 878 & 1147 & 1203 & 1459 \\
\hline 27 & 41 & 68 & 108 & 179 & 251 & 381 & 515 & 711 & 939 & 1027 \\
\hline
\end{tabular}

\begin{tabular}{l|l}
68 & 77 \\
\hline 39 & 44 \\
\hline
\end{tabular}

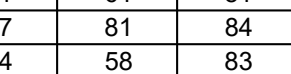

\begin{tabular}{|c|c|c|c|c|c|c|}
89 & 94 & 100 & 104 & 108 & 111 & 117 \\
\hline 111 & 164 & 242 & 392 & 607 & 686 & 976 \\
\hline
\end{tabular}

\begin{tabular}{|c|c|c|c|c|c|c|c|c|c|c|}
94 & 146 & 209 & 299 & 402 & 551 & 744 & 977 & 1165 & 1497 & 2000 \\
\hline 23 & 37 & 50 & 82 & 130 & 228 & 350 & 519 & 730 & 886 & 1051 \\
\hline
\end{tabular}

\begin{tabular}{|c|c|c|c|c|c|c|c|c|c|c|c|c|}
300 & 364 & 50 & 82 & 130 & 228 & 350 & 519 & 730 & 886 & 1051 \\
\hline
\end{tabular}

\begin{tabular}{c|c|c|c|}
147 & 176 & 217 & 258 \\
\hline 17 & 27 & 38 & 49 \\
\hline
\end{tabular}

\begin{tabular}{|l|l|l|l|l|l|l|}
295 & 334 & 347 & 387 & 440 & 457 & 526 \\
\hline
\end{tabular}

\begin{tabular}{|c|c|c|c|}
17 & 27 & 38 & 49 \\
\hline 92 & 132 & 213 & 345 \\
\hline 200 & 282 & 319 & 363
\end{tabular}

$80+125$

\begin{tabular}{|c|c|c|c|c|c|c|c|c|c|}
132 & 213 & 345 & 503 & 735 & 1049 & 1494 & 1799 & 2154 & 2826 \\
\hline 282 & 319 & 363 & 423 & 511 & 580 & 615 & 737 & 819 & 986 \\
\hline 145 & 211 & 250 & 287 & 370 & 456 & 543 & 581 & 620 & \\
\hline
\end{tabular}

\begin{tabular}{l|c|c|c|}
\hline 94 & 145 & 211 & 250 \\
\hline 50 & 75 & 100 & 130 \\
\hline
\end{tabular}

\begin{tabular}{l|l|l|}
423 & 511 & 580 \\
\hline 287 & 370 & 456 \\
\hline
\end{tabular}

\begin{tabular}{l|c|c|c|}
50 & 75 & 100 & 130 \\
\hline 65 & 70 & 74 & 78
\end{tabular}

\begin{tabular}{|l|l|l|l|l|l|l|}
287 & 370 & 456 & 543 & 581 & 620 & \\
\hline 200 & 315 & 404 & 535 & 685 & 782 & 900 \\
\hline
\end{tabular}

\begin{tabular}{|c|c|c|c|c|c|c|c|c|c|c|}
7 & 9 & 11 & 78 & 81 & 82 & 87 & 89 & 93 & 98 & 102 \\
\hline
\end{tabular}

\begin{tabular}{|c|c|c|c|c|c|c|c|c|c|c|}
\hline 7 & 9 & 11 & 13 & 15 & 19 & 20 & 22 & 24 & 26 & 28 \\
\hline 6 & 11 & 22 & 48 & 66 & 150 & 225 & 375 & 600 & 726 & 932 \\
\hline 100 & 146 & 209 & 299 & 402 & 551 & 744 & 977 & 1165 & 1497 & 2000 \\
\hline
\end{tabular}

\begin{tabular}{|c|c|c|c|c|c|c|c|c|c|c|c|} 
& 12 & 22 & 32 & 62 & 106 & 170 & 250 & 368 & 509 & 697 & 890 \\
\hline
\end{tabular}

$\mathrm{O}: \mathrm{CH} 3: \mathrm{D}$

$\cdot \mathrm{F} \cdot \mathrm{D}$

o:CF3:D

$\mathrm{O:NO2: \textrm {D }}$

$\mathrm{m}: \mathrm{NH} 2: \mathrm{E}$

$\mathrm{m}: \mathrm{OCH} 3: \mathrm{E}$

$\mathrm{m}: \mathrm{CH} 3: \mathrm{E}$

\begin{tabular}{|l|l|l|l|}
\hline$m: F: E$ & 35 & 44 \\
\hline$m: C F 3: E$ & 62 & 78 \\
\hline
\end{tabular}

m:NO2:E

$\mathrm{O}: \mathrm{NH2: \textrm {E }}$

$\mathrm{O}: \mathrm{CH} 3: \mathrm{E}$

\begin{tabular}{|l|c|c|c|c|c|c|c|c|c|c|c|}
\hline $\mathrm{m}: \mathrm{NO} 2: \mathrm{D}$ & 6 & 9 & 11 & 14 & 18 & 26 & 31 & 49 & 58 & 67 & 76 \\
\hline $\mathrm{o}: \mathrm{CH} 3: \mathrm{D}$ & 40 & 43 & 47 & 54 & 62 & 65 & 69 & 79 & 85 & 94 & 101 \\
\hline $\mathrm{o}: \mathrm{F}: \mathrm{D}$ & 7 & 11 & 17 & 24 & 32 & 50 & 67 & 86 & 115 & 132 & 154 \\
\hline o:CF3:D & 11 & 14 & 17 & 19 & 25 & 28 & 33 & 36 & 38 & 40 & 42 \\
\hline $\mathrm{o}: \mathrm{NO} 2: \mathrm{D}$ & 68 & 77 & 81 & 84 & 89 & 94 & 100 & 104 & 108 & 111 & 117 \\
\hline $\mathrm{m}: \mathrm{NH} 2: \mathrm{E}$ & 10 & 23 & 31 & 42 & 88 & 173 & 244 & 488 & 758 & 953 & 1115 \\
\hline $\mathrm{m}: \mathrm{OCH} 3: \mathrm{E}$ & 16 & 24 & 50 & 88 & 162 & 269 & 434 & 600 & 818 & 1048 & 2002 \\
\hline $\mathrm{m}: \mathrm{CH} 3: \mathrm{E}$ & 78 & 86 & 125 & 160 & 200 & 260 & 368 & 507 & 649 & 785 & 885 \\
\hline $\mathrm{m}: \mathrm{F}: \mathrm{E}$ & 35 & 44 & 71 & 106 & 166 & 282 & 429 & 586 & 707 & 933 & 1160 \\
\hline $\mathrm{m}: \mathrm{CF} 3: \mathrm{E}$ & 62 & 78 & 115 & 155 & 227 & 338 & 509 & 739 & 1116 & 1604 & 2147 \\
\hline $\mathrm{m}: \mathrm{NO2}: \mathrm{E}$ & 40 & 68 & 98 & 115 & 173 & 273 & 387 & 600 & 886 & 1289 & 1376 \\
\hline $\mathrm{o}: \mathrm{NH} 2 \mathrm{E}$ & 175 & 242 & 280 & 340 & 400 & 507 & 600 & 872 & 1058 & 1249 & 1334 \\
\hline o:CH3:E & 3 & 4 & 6 & 9 & 12 & 17 & 25 & 31 & 33 & 43 & 68 \\
\hline o:F:E & 12 & 18 & 24 & 30 & 39 & 45 & 57 & 68 & 81 & 93 & 102 \\
\hline Temperature (K) & 277.50 & 280.73 & 283.96 & 287.19 & 290.42 & 293.65 & 296.88 & 300.11 & 303.34 & 306.57 & 309.80 \\
\hline o:CH3:A & 172 & 188 & 214 & 258 & 292 & 378 & 438 & 568 & 695 & 912 & 1159 \\
\hline o:F:A & 9 & 12 & 16 & 19 & 22 & 27 & 28 & 34 & 36 & 46 & 48 \\
\hline o:CF3:A & 11 & 25 & 32 & 49 & 32 & 34 & 36 & 38.9 & 43.1 & 48.2 & 65.9 \\
\hline m:CH3:A & 5 & 5 & 19 & & 33 & 64.6 & 79 & 109 & 138 & 183 & 236 \\
\hline m:F:A & 71 & 84 & 93 & 98 & 120 & 121 & 133 & 145 & 181 & 223 & 270
\end{tabular}

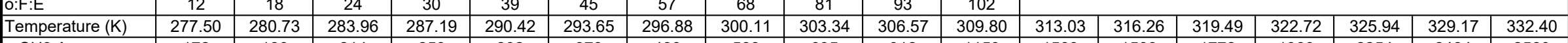
$\mathrm{o}: \mathrm{CH} 3: \mathrm{A}$

O.F.A

o:CF3:A

$\mathrm{m}: \mathrm{CH} 3: \mathrm{A}$

m:F:A 


\begin{tabular}{|c|c|c|c|c|c|c|c|c|c|c|c|c|c|c|c|c|c|c|}
\hline m:CF3:A & 38.9 & 42.9 & 49.9 & 68.9 & 80.9 & 97.9 & 107.9 & 131.9 & 155.9 & 183.9 & 182.9 & 245.9 & 279.9 & 326.9 & 405.9 & 507.9 & 665.9 & 892.9 \\
\hline$m: N O 2: A$ & & & 13 & 23 & 32 & 47 & 51 & 67 & 84 & 109 & 138 & 191 & 251 & 334 & 400 & 662 & 662 & 847 \\
\hline $\mathrm{m}: \mathrm{OCH} 3: \mathrm{A}$ & 10.9 & 24.9 & 76 & 91.9 & 121.9 & 168.9 & 223.9 & 291.9 & 349.9 & 483.9 & 633.9 & 811.9 & 1042.9 & 1335.9 & 1889.9 & 2098.9 & 2076.9 & 2078.9 \\
\hline $\mathrm{O}: \mathrm{OCH} 3: \mathrm{A}$ & 56 & 59 & 57 & 62 & 60 & 66 & 67 & 68 & 69 & 71 & 72 & 75 & 78 & 80 & 84 & 86 & 90 & 99 \\
\hline $\mathrm{H}: \mathrm{A}$ & 26 & 40 & 50 & 55 & 56 & 73 & 90 & 107 & 121 & 142 & 185 & 238 & 314 & 404 & 524 & 665 & 823 & 921 \\
\hline o:NO2:A & 235 & 346 & 472 & 751 & 1060 & 1518 & 2142 & 3200 & 4500 & 6800 & 9700 & 13000 & 18000 & 21000 & 24000 & 27000 & 30000 & 33000 \\
\hline
\end{tabular}




\begin{tabular}{|c|c|c|c|c|}
\hline \multicolumn{5}{|c|}{ Table S2 } \\
\hline Molecule & $\Delta \mathrm{H}(\mathrm{kJ} / \mathrm{mol})$ & $\Delta \mathrm{He}(\mathrm{kJ} / \mathrm{mol})$ & $\Delta \mathrm{S}(\mathrm{J} / \mathrm{mol} . \mathrm{K})$ & $\Delta \mathrm{Se}(\mathrm{J} / \mathrm{mol} . \mathrm{K})$ \\
\hline $\mathrm{o:OCH} 3: \mathrm{A}$ & 4.58 & 0.32 & -190.80 & 1.07 \\
\hline $0: C F 3: A$ & 29.76 & 3.14 & -110.84 & 10.40 \\
\hline $\mathrm{O}: \mathrm{F}: \mathrm{A}$ & 34.06 & 0.80 & -98.50 & 2.66 \\
\hline$m: F: A$ & 35.71 & 2.26 & -78.00 & 7.48 \\
\hline$m: C F 3: A$ & 37.95 & 1.20 & -73.91 & 3.96 \\
\hline $\mathrm{O}: \mathrm{CH} 3: \mathrm{A}$ & 39.24 & 1.10 & -57.33 & 3.65 \\
\hline$: \mathrm{H}: \mathrm{A}$ & 45.62 & 1.54 & -49.29 & 5.12 \\
\hline$m: N O 2: A$ & 62.37 & 1.43 & 1.70 & 4.66 \\
\hline $\mathrm{m}: \mathrm{OCH} 3: \mathrm{A}$ & 68.56 & 3.50 & 32.51 & 11.60 \\
\hline o:NO2:A & 71.53 & 1.99 & 63.54 & 6.60 \\
\hline $\mathrm{m}: \mathrm{CH} 3: \mathrm{A}$ & 78.65 & 3.46 & 58.21 & 11.37 \\
\hline $\mathrm{o}: \mathrm{CF} 3: \mathrm{B}$ & 4.73 & 0.27 & -188.08 & 0.90 \\
\hline$m: N O 2: B$ & 13.59 & 0.72 & -156.57 & 2.37 \\
\hline$m: C F 3: B$ & 15.10 & 0.40 & -144.29 & 1.31 \\
\hline$m: C H 3: B$ & 21.61 & 0.67 & -111.99 & 2.22 \\
\hline $\mathrm{O}: \mathrm{F}: \mathrm{B}$ & 27.59 & 1.25 & -110.51 & 4.13 \\
\hline $\mathrm{m}: \mathrm{OCH} 3: \mathrm{B}$ & 27.96 & 0.77 & -90.69 & 2.53 \\
\hline$m: F: B$ & 35.77 & 0.81 & -67.82 & 2.68 \\
\hline $\mathrm{o}: \mathrm{OCH} 3: \mathrm{B}$ & 38.34 & 1.52 & -64.53 & 5.01 \\
\hline $\mathrm{o}: \mathrm{CH} 3: \mathrm{B}$ & 51.57 & 1.61 & -26.67 & 5.32 \\
\hline $\mathrm{m}: \mathrm{NH} 2: \mathrm{B}$ & 56.99 & 2.64 & -28.70 & 8.73 \\
\hline $\mathrm{m}: \mathrm{H}: \mathrm{B}$ & 72.90 & 2.12 & 32.55 & 7.00 \\
\hline $\mathrm{o:NH2:B}$ & 74.18 & 1.61 & 42.40 & 5.31 \\
\hline $\mathrm{O}: \mathrm{F}: \mathrm{C}$ & 3.46 & 0.20 & -193.20 & 0.66 \\
\hline$m: F: C$ & 13.75 & 0.63 & -143.69 & 2.09 \\
\hline$m: C F 3: C$ & 14.77 & 0.80 & -145.14 & 2.64 \\
\hline o:NO2:C & 16.85 & 1.06 & -162.66 & 3.51 \\
\hline $\mathrm{o:NH2:C}$ & 18.38 & 0.69 & -129.47 & 2.29 \\
\hline $\mathrm{o}: \mathrm{OCH} 3: \mathrm{C}$ & 26.11 & 1.86 & -106.96 & 6.19 \\
\hline $\mathrm{m}: \mathrm{OCH} 3: \mathrm{C}$ & 39.94 & 0.77 & -58.05 & 2.53 \\
\hline $\mathrm{o}: \mathrm{CH} 3: \mathrm{C}$ & 40.49 & 1.45 & -61.76 & 4.79 \\
\hline$m: N H 2: C$ & 46.44 & 2.10 & -45.18 & 6.95 \\
\hline $\mathrm{H}: \mathrm{C}$ & 46.72 & 2.12 & -33.73 & 6.99 \\
\hline $\mathrm{m}: \mathrm{CH} 3: \mathrm{C}$ & 55.23 & 1.73 & -15.64 & 5.69 \\
\hline$m: N O 2: C$ & 57.34 & 1.76 & -11.70 & 5.81 \\
\hline o:NO2:D & 4.73 & 0.27 & -188.08 & 0.90 \\
\hline $\mathrm{o}: \mathrm{CH} 3: \mathrm{D}$ & 10.94 & 0.33 & -170.67 & 1.09 \\
\hline $\mathrm{o}: \mathrm{CF} 3: \mathrm{D}$ & 16.89 & 1.23 & -158.84 & 4.07 \\
\hline$m: C F 3: D$ & 28.78 & 0.83 & -124.24 & 2.75 \\
\hline$m: N O 2: D$ & 34.72 & 1.12 & -100.39 & 3.70 \\
\hline $\mathrm{m}: \mathrm{OCH} 3: \mathrm{D}$ & 39.52 & 0.62 & -59.40 & 2.06 \\
\hline $\mathrm{O}: \mathrm{F}: \mathrm{D}$ & 42.21 & 1.43 & -71.19 & 4.71 \\
\hline $\mathrm{m}: \mathrm{F}: \mathrm{D}$ & 48.01 & 1.85 & -38.94 & 6.09 \\
\hline$m: C H 3: D$ & 59.90 & 1.66 & -3.13 & 5.48 \\
\hline
\end{tabular}




\begin{tabular}{|l|c|c|c|c|}
\hline $\mathrm{m}: \mathrm{NH} 2: \mathrm{D}$ & 71.86 & 2.53 & 34.42 & 8.34 \\
\hline $\mathrm{o}: \mathrm{NH} 2: \mathrm{E}$ & 27.19 & 1.00 & -99.74 & 3.31 \\
\hline $\mathrm{o}: \mathrm{F}: \mathrm{E}$ & 27.31 & 1.10 & -120.17 & 3.64 \\
\hline $\mathrm{m}: \mathrm{CH}: \mathrm{E}$ & 34.76 & 1.09 & -80.21 & 3.59 \\
\hline $\mathrm{o}: \mathrm{CH}: \mathrm{E}$ & 40.82 & 1.33 & -84.21 & 4.38 \\
\hline $\mathrm{m}: \mathrm{NO} 2: \mathrm{E}$ & 49.39 & 1.51 & -32.06 & 4.99 \\
\hline $\mathrm{m}: \mathrm{CF} 3: \mathrm{E}$ & 49.79 & 1.44 & -28.57 & 4.75 \\
\hline $\mathrm{m}: \mathrm{F}: \mathrm{E}$ & 50.64 & 1.68 & -29.18 & 5.55 \\
\hline $\mathrm{m}: \mathrm{OCH} 3: \mathrm{E}$ & 65.57 & 2.01 & 19.15 & 6.64 \\
\hline $\mathrm{m}: \mathrm{NH} 2: \mathrm{E}$ & 67.41 & 2.48 & 21.96 & 8.20 \\
\hline
\end{tabular}



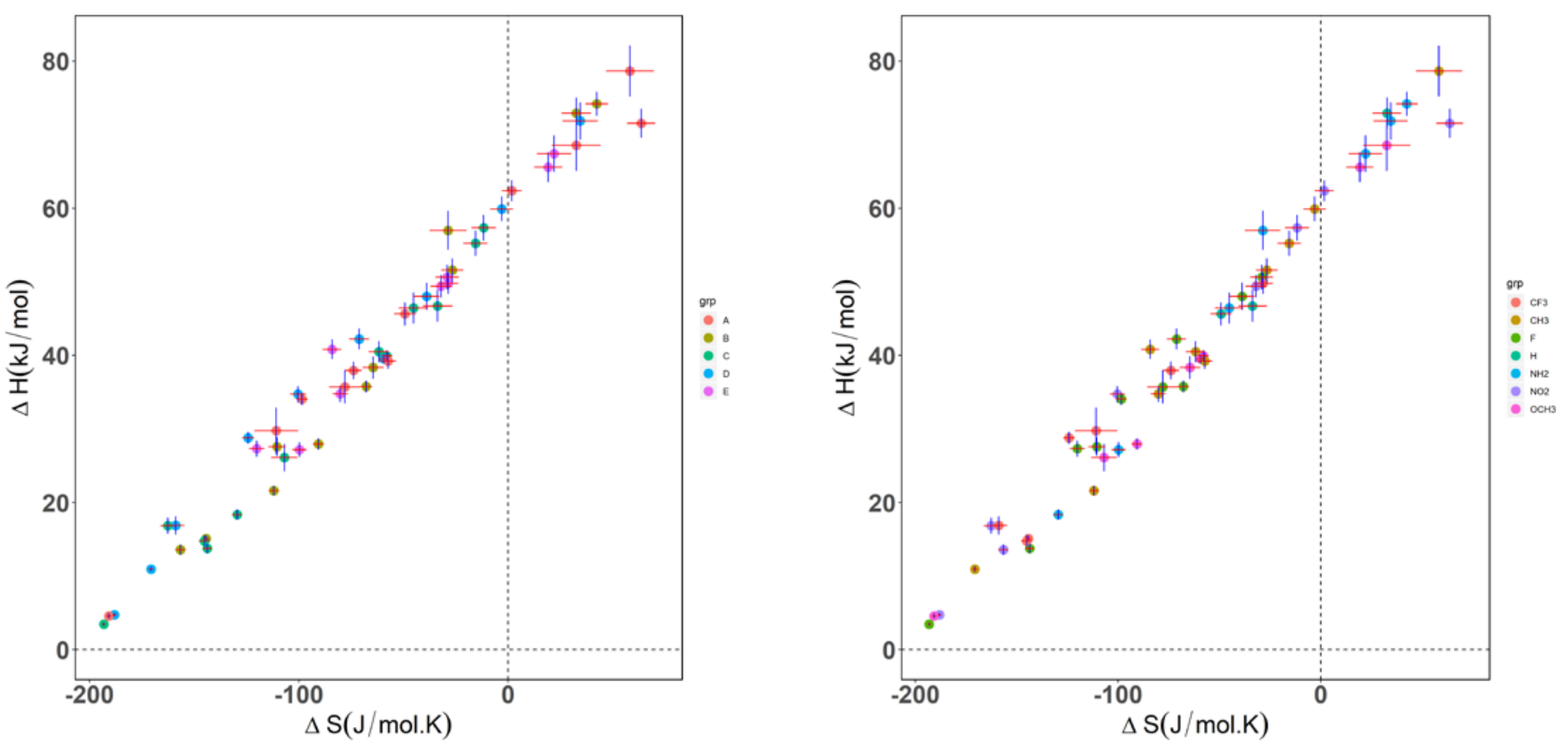

Figure S1: EEC plot for the sub-groups.

(a) As a function of groups on A, B, C, D, and E and (b) as a function of the functional groups (marked on the right) 
Figure S2: NMR $\left({ }^{1} \mathrm{H}\right.$ and $\left.{ }^{13} \mathrm{C}\right)$ spectra of all the molecules. Molecular structures are shown at the top of each spectra 
Figure S2

SB
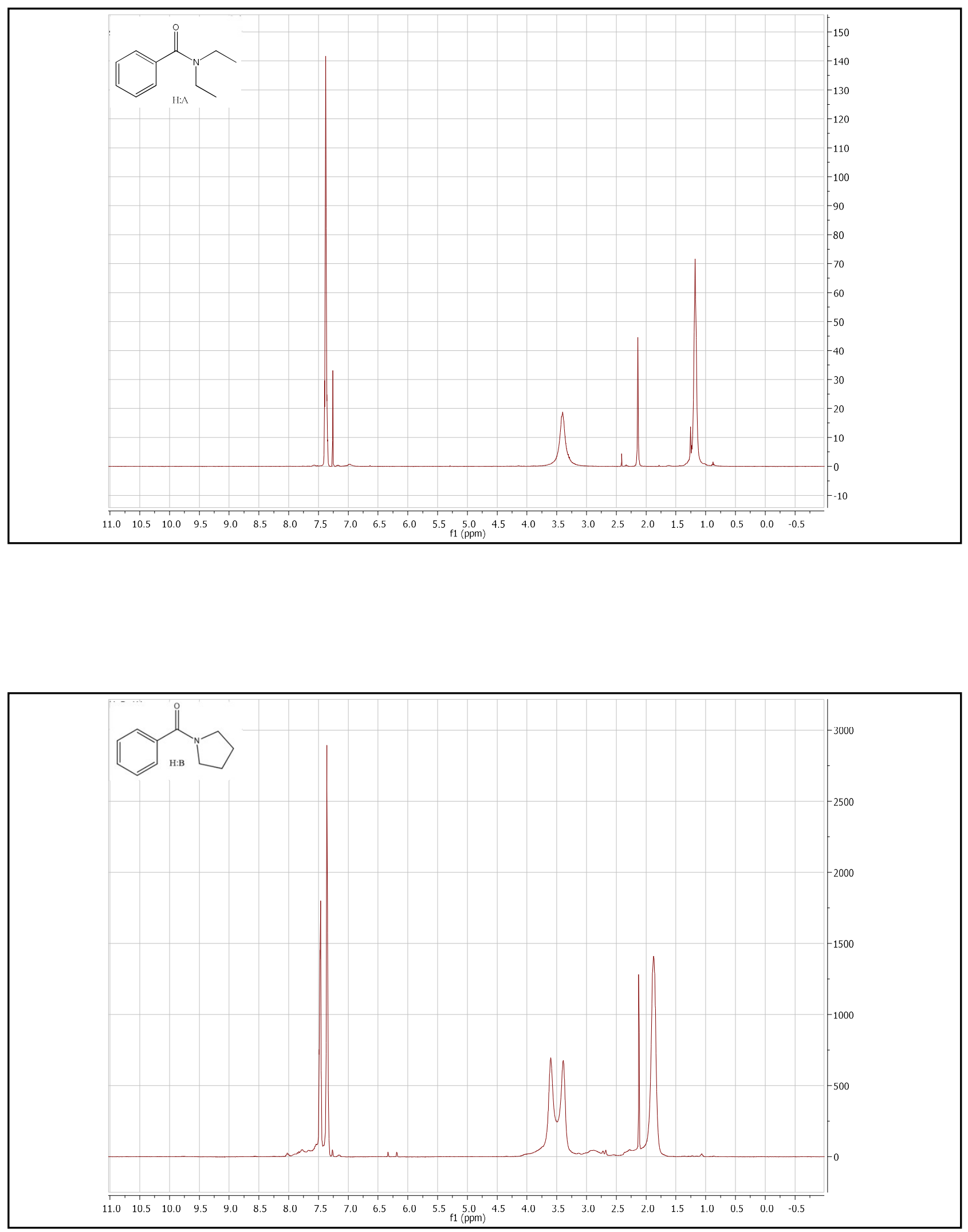

1 
Figure S2

Sg
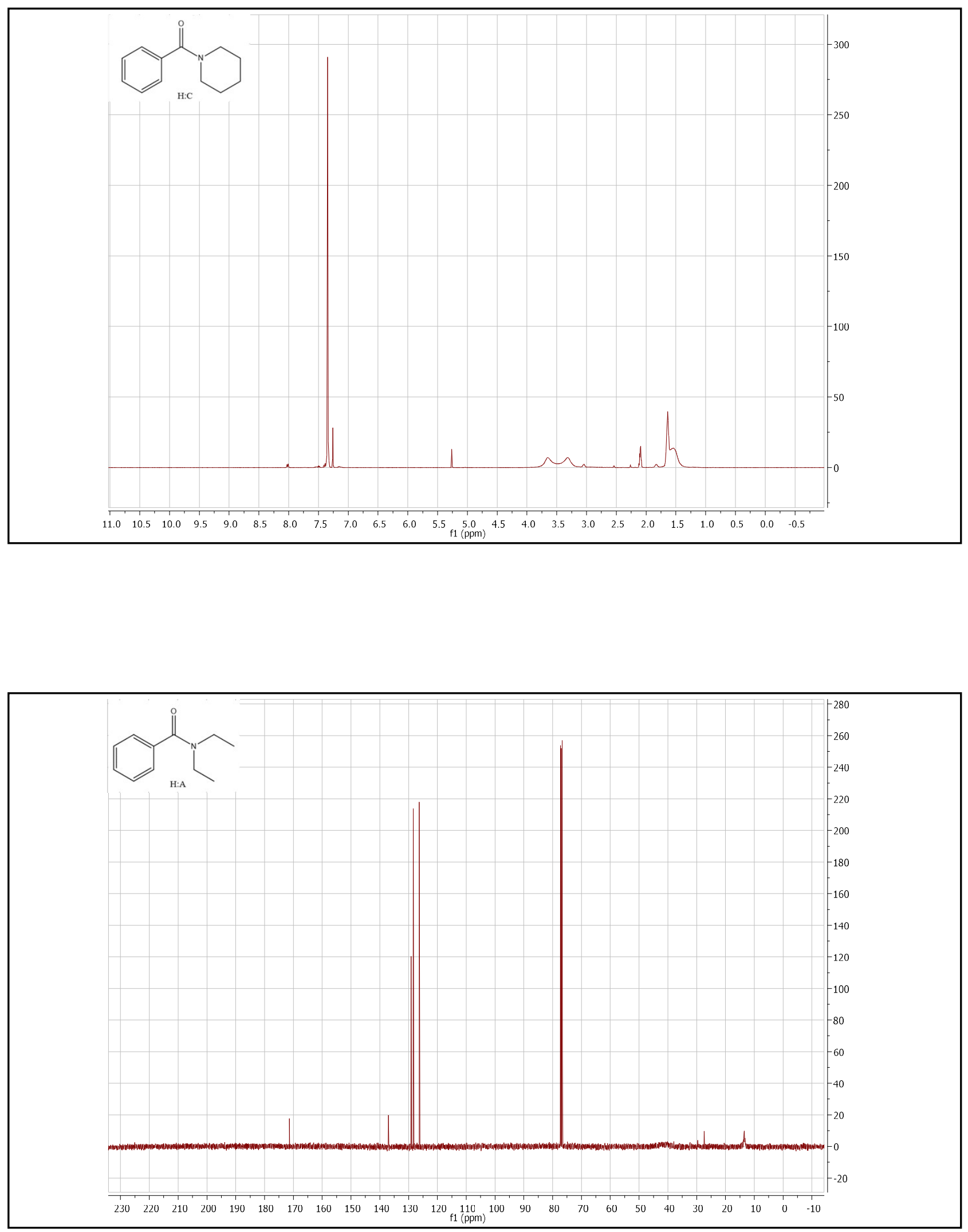

2 
Figure S2

S10
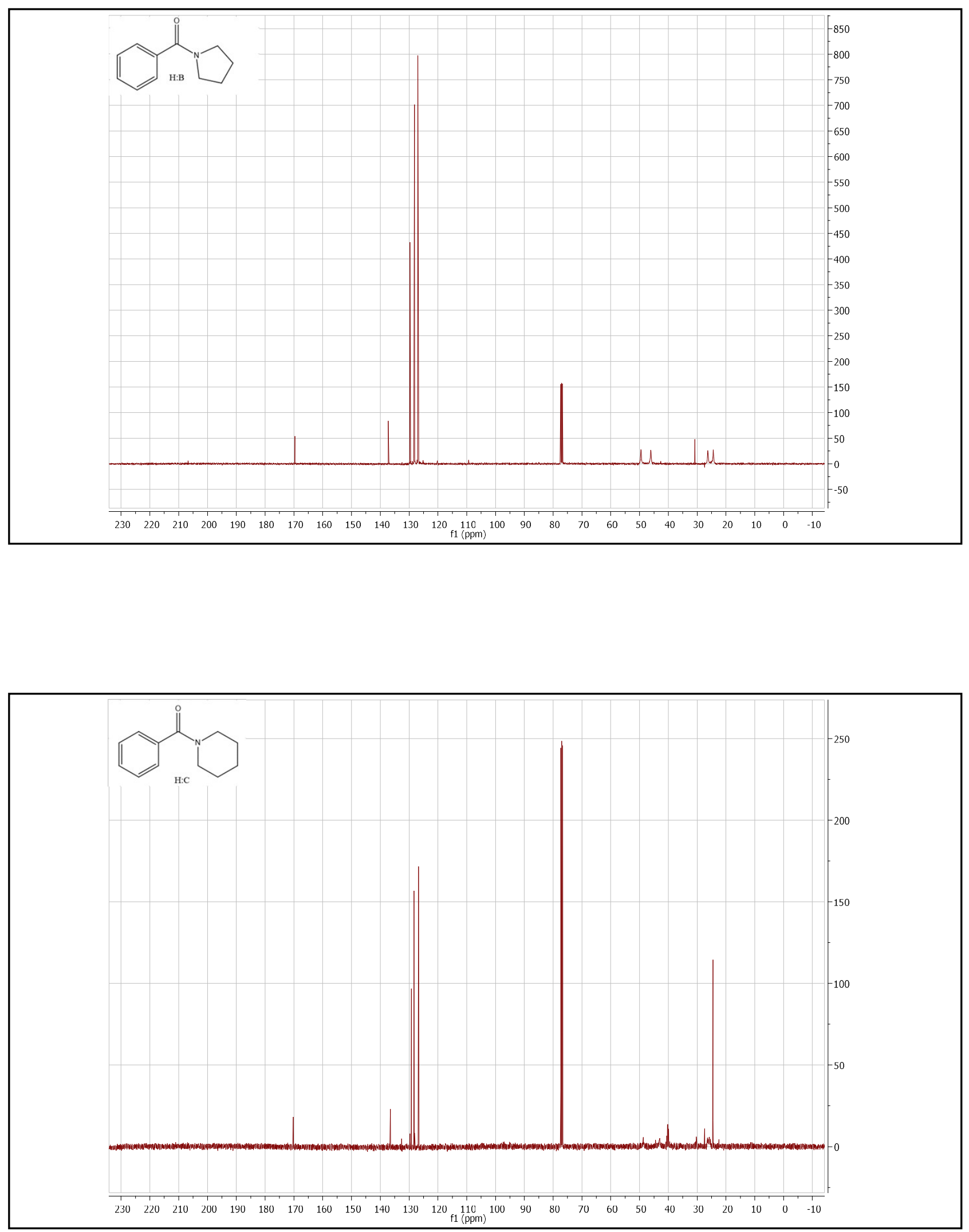

3 
Figure S2

S11
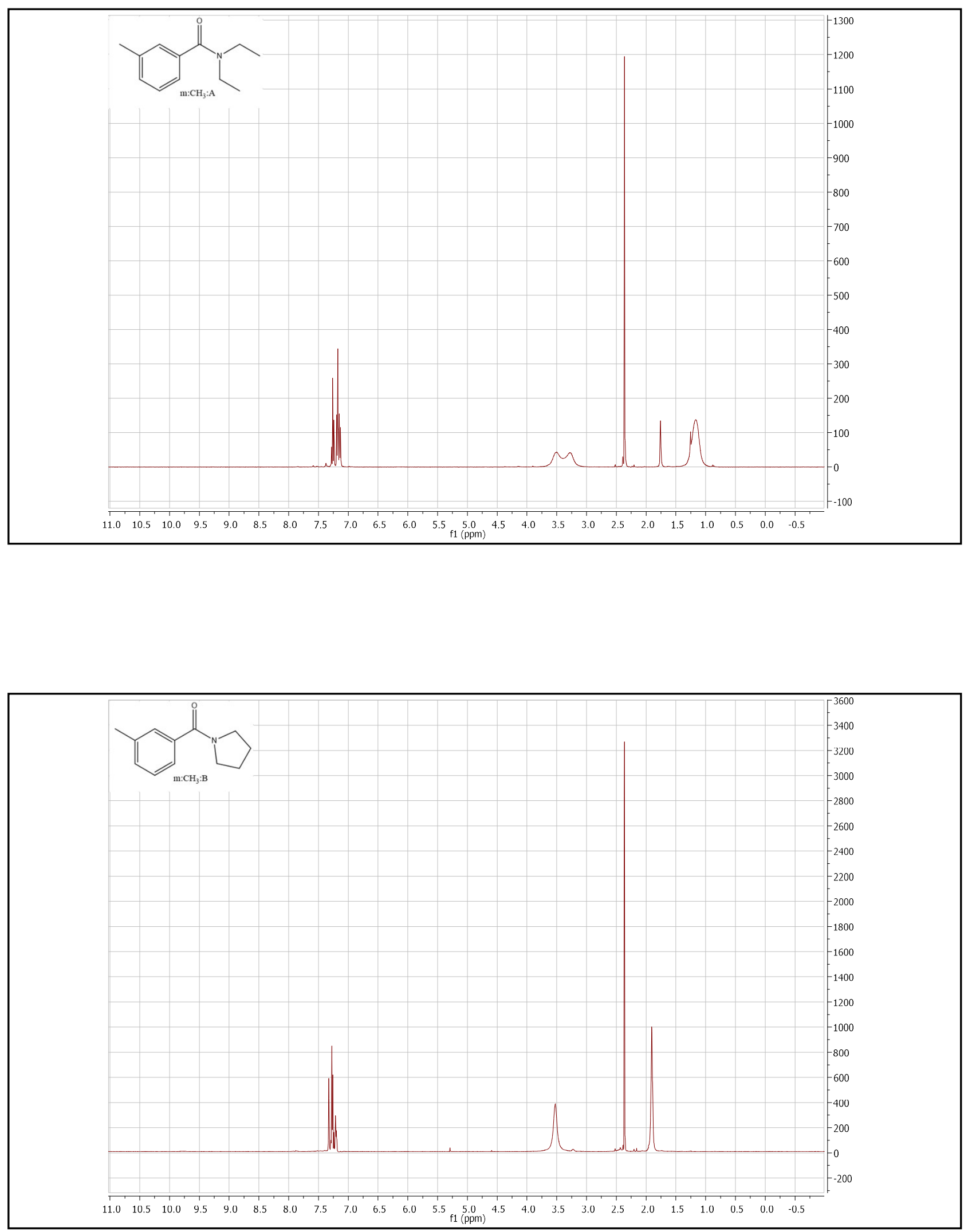

4 
Figure S2

$\mathrm{S} 12$
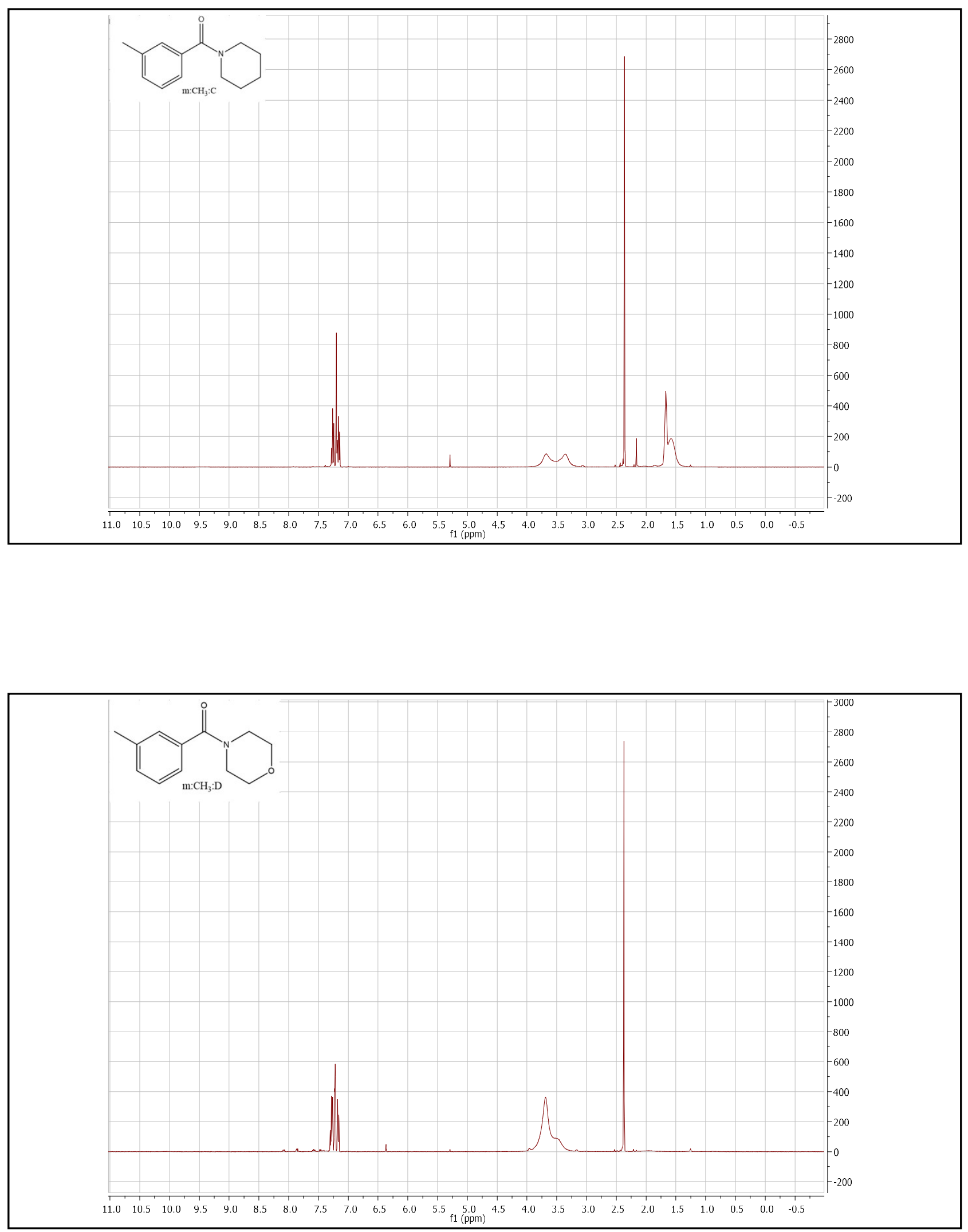

5 
Figure S2

$\mathrm{S} 13$
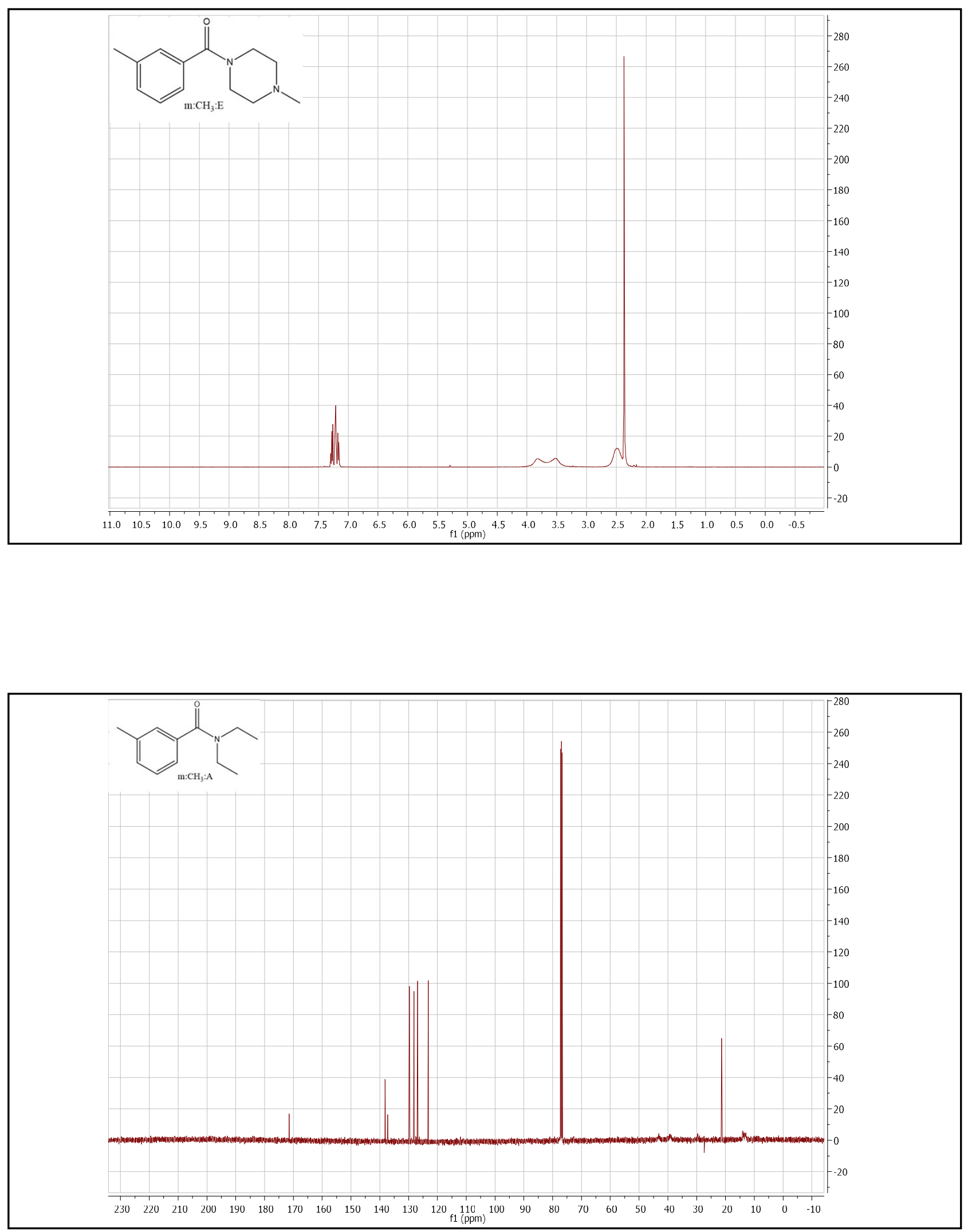

6 
Figure S2

S14
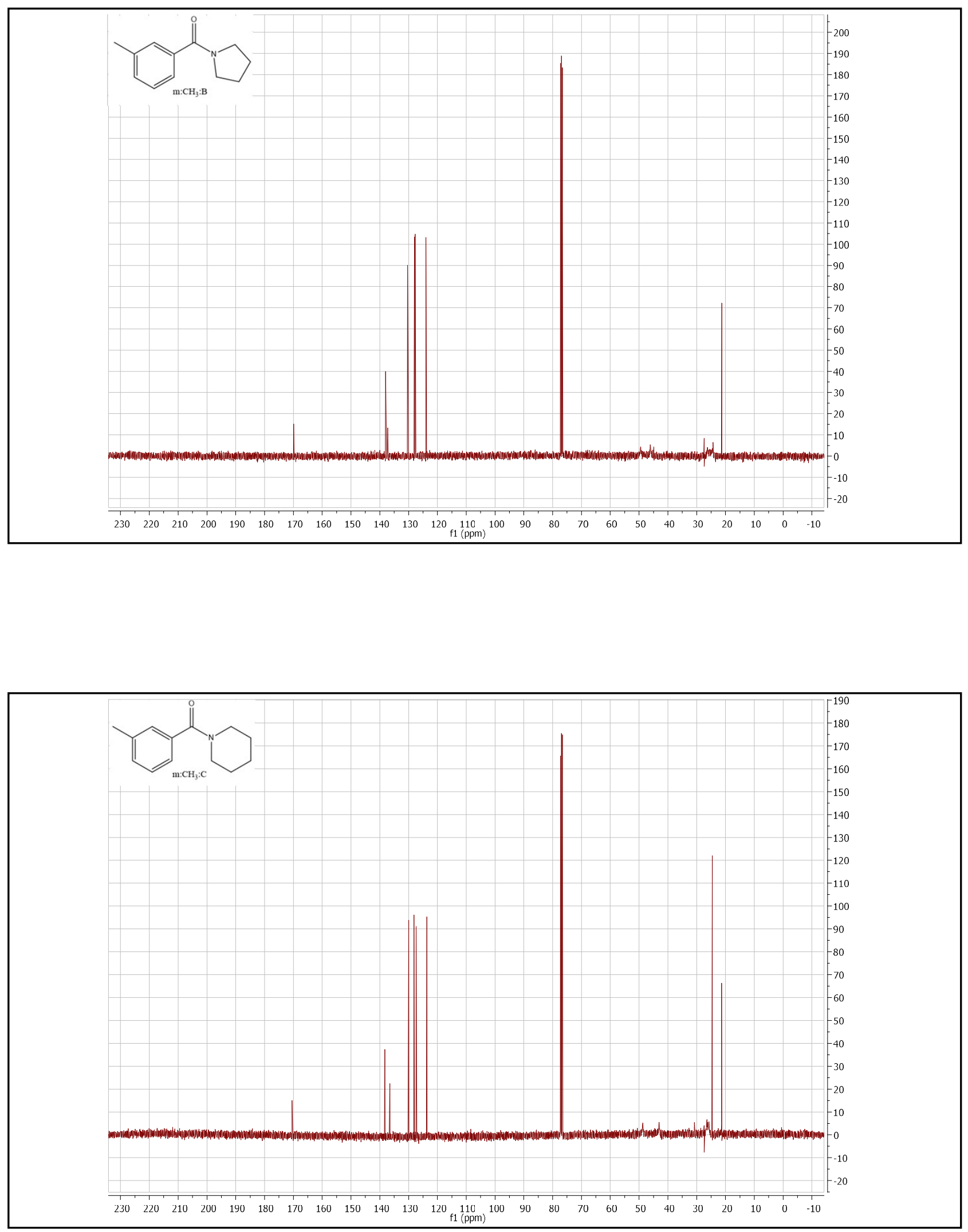

7 


$$
\frac{51}{41}
$$


Figure S2

S16
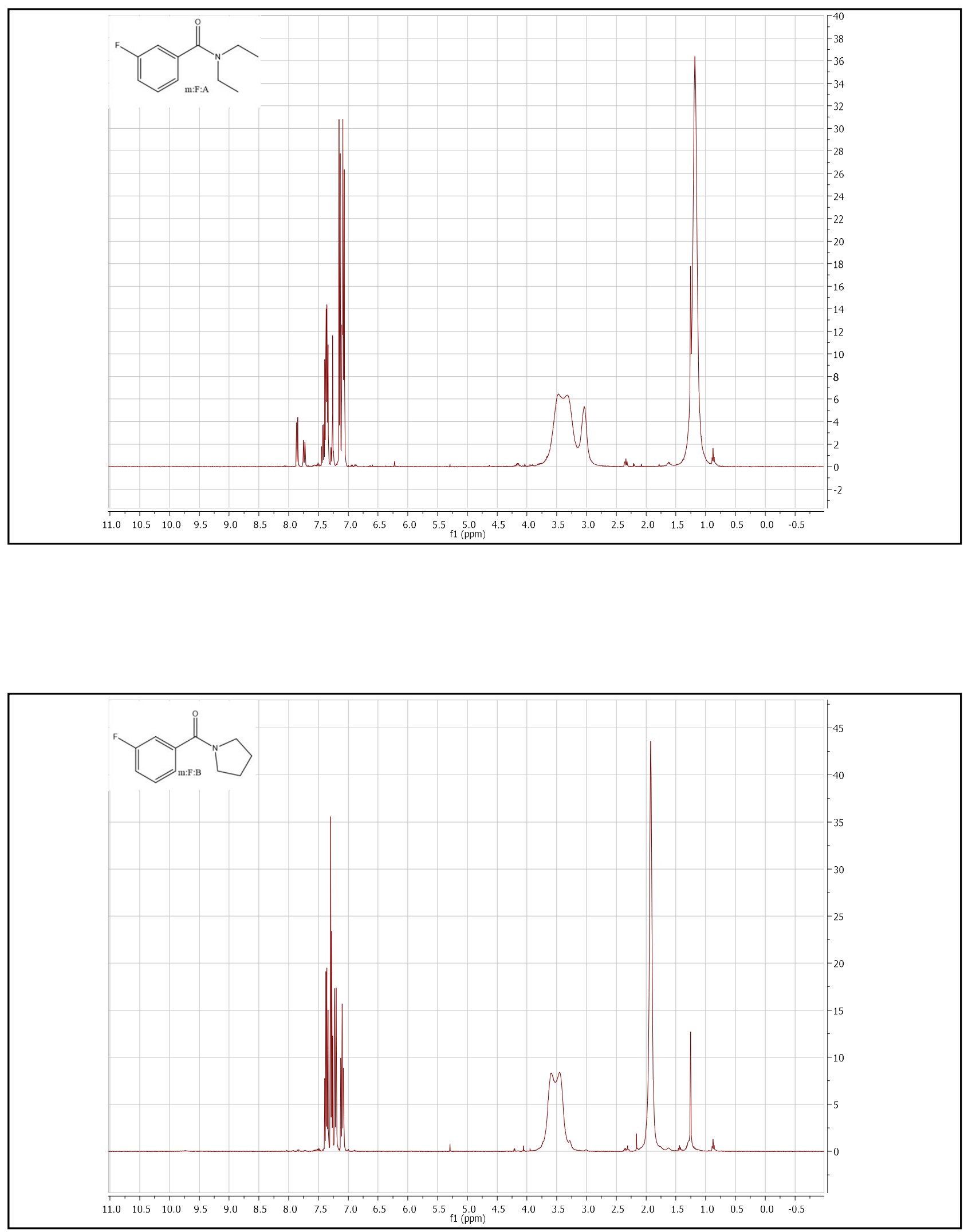

9 
Figure S2

S17
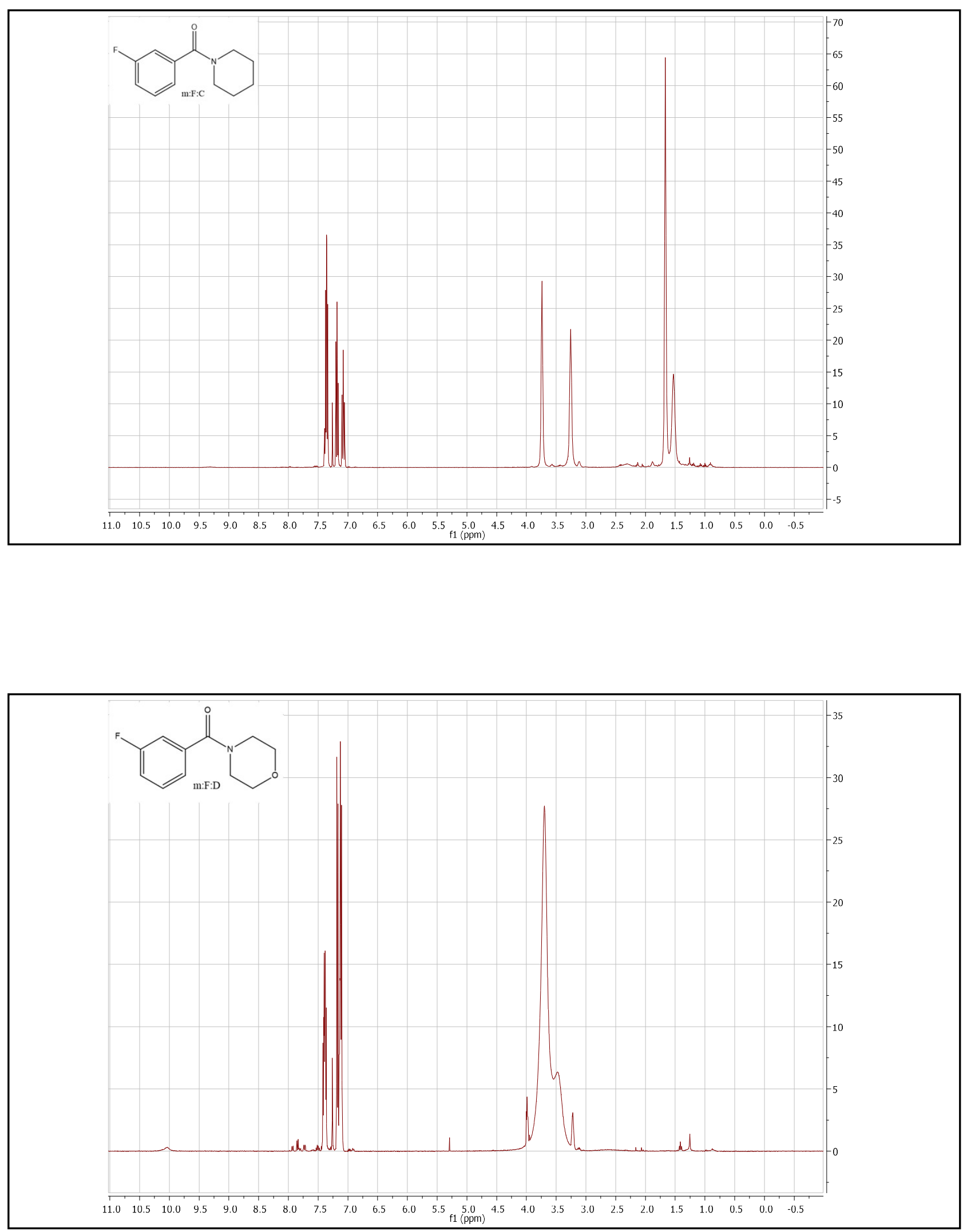

10 
Figure S2

S18
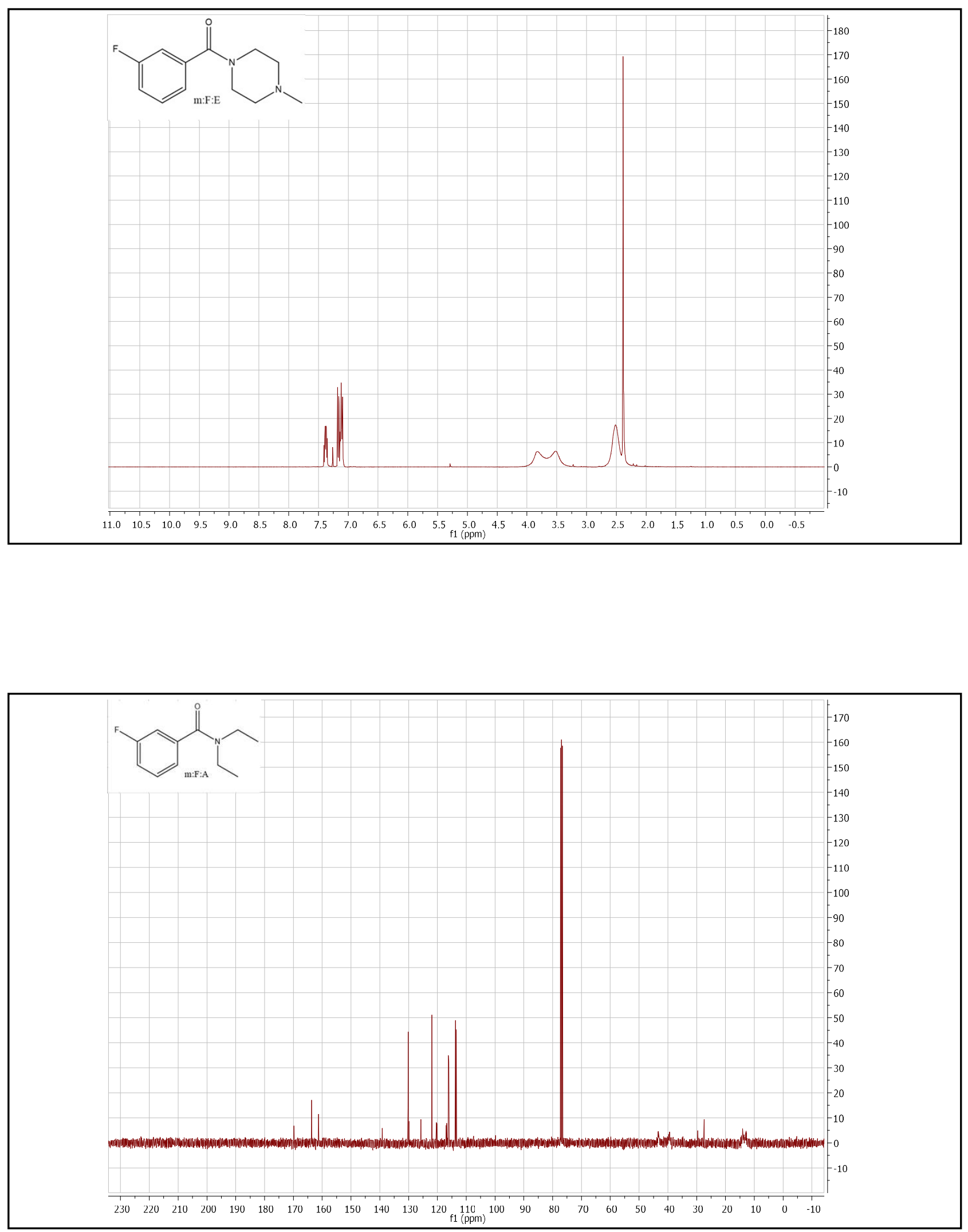

11 
Figure S2

S19
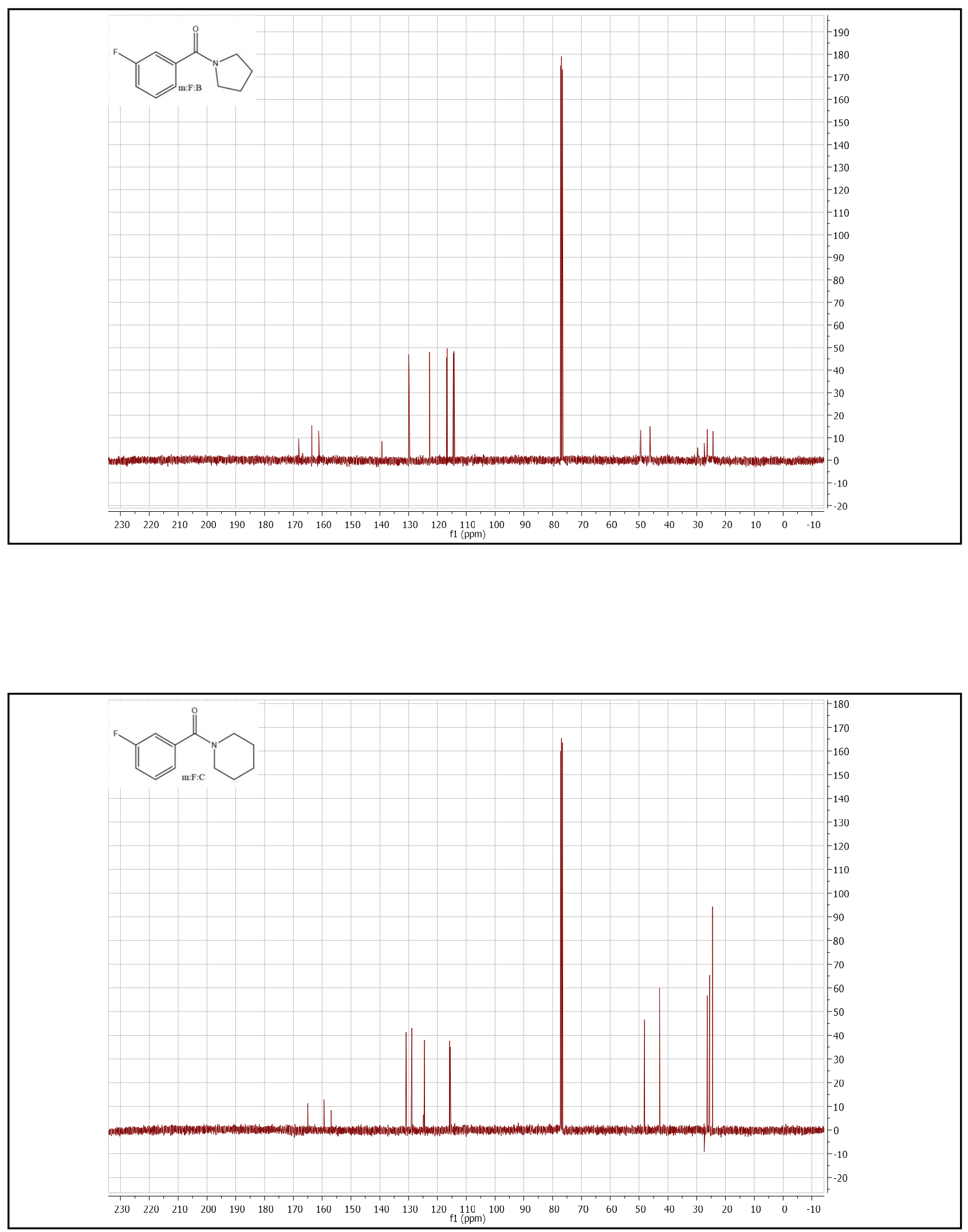

12 
Figure S2

S20
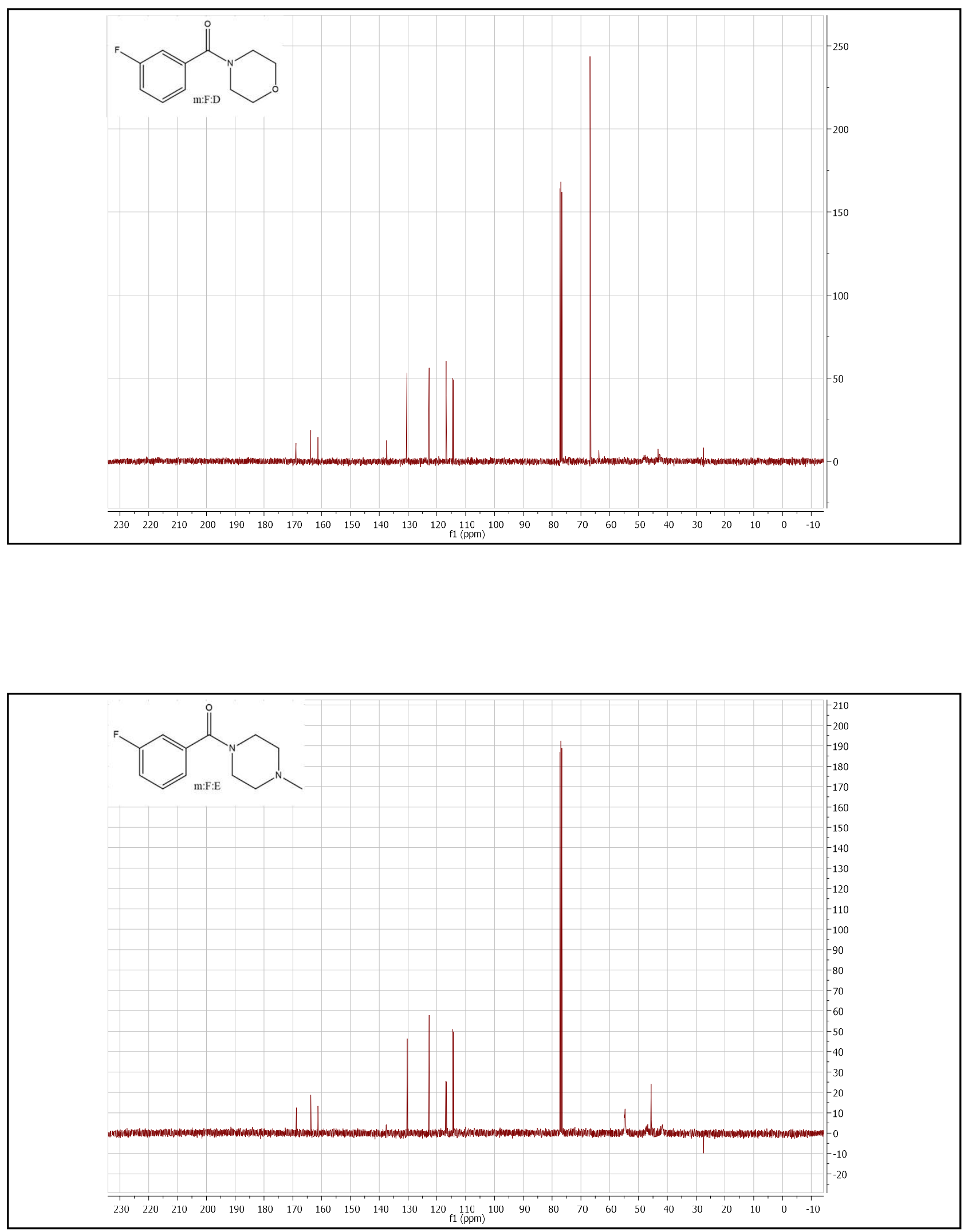

13 


\section{Figure S2}
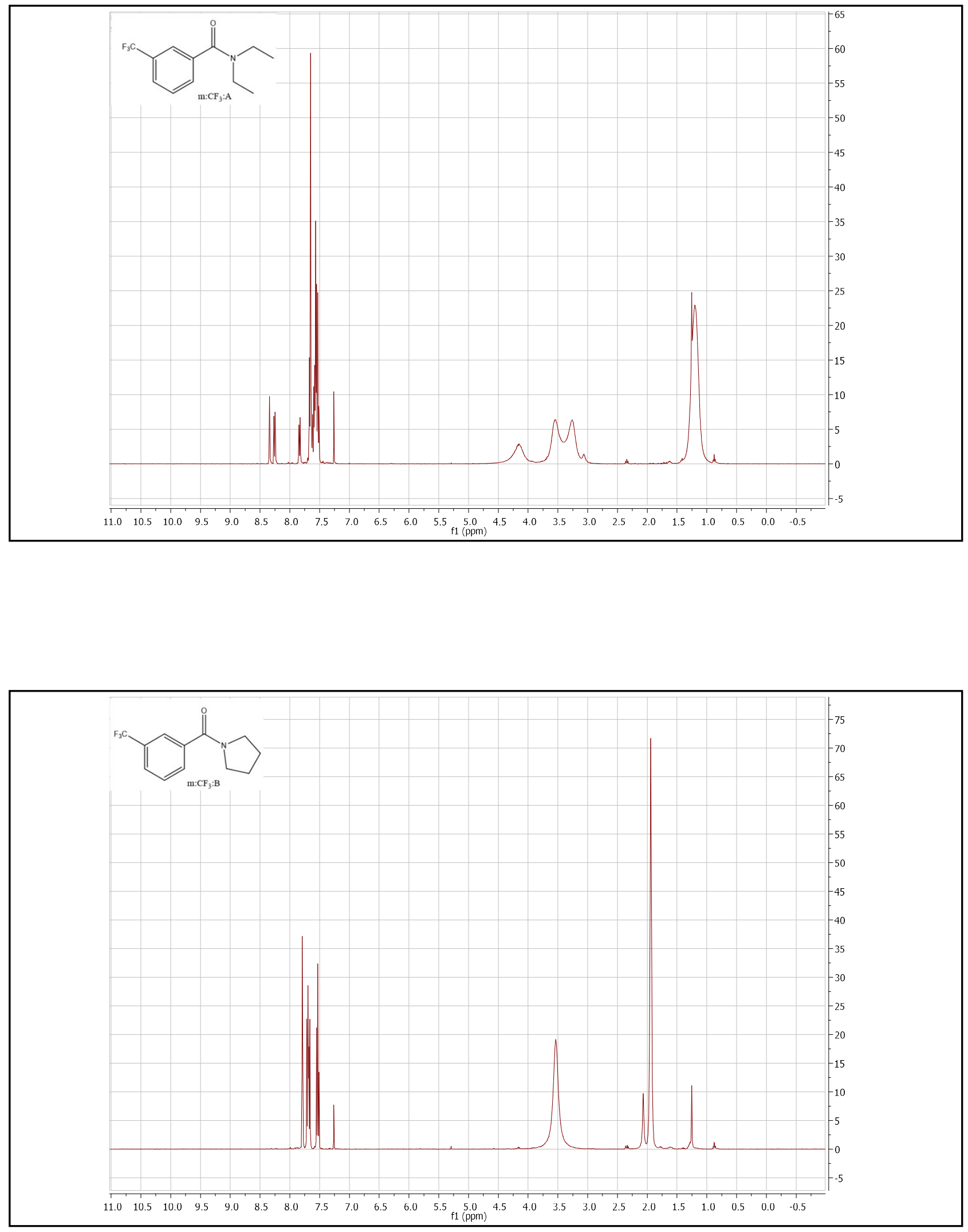
Figure S2

S22
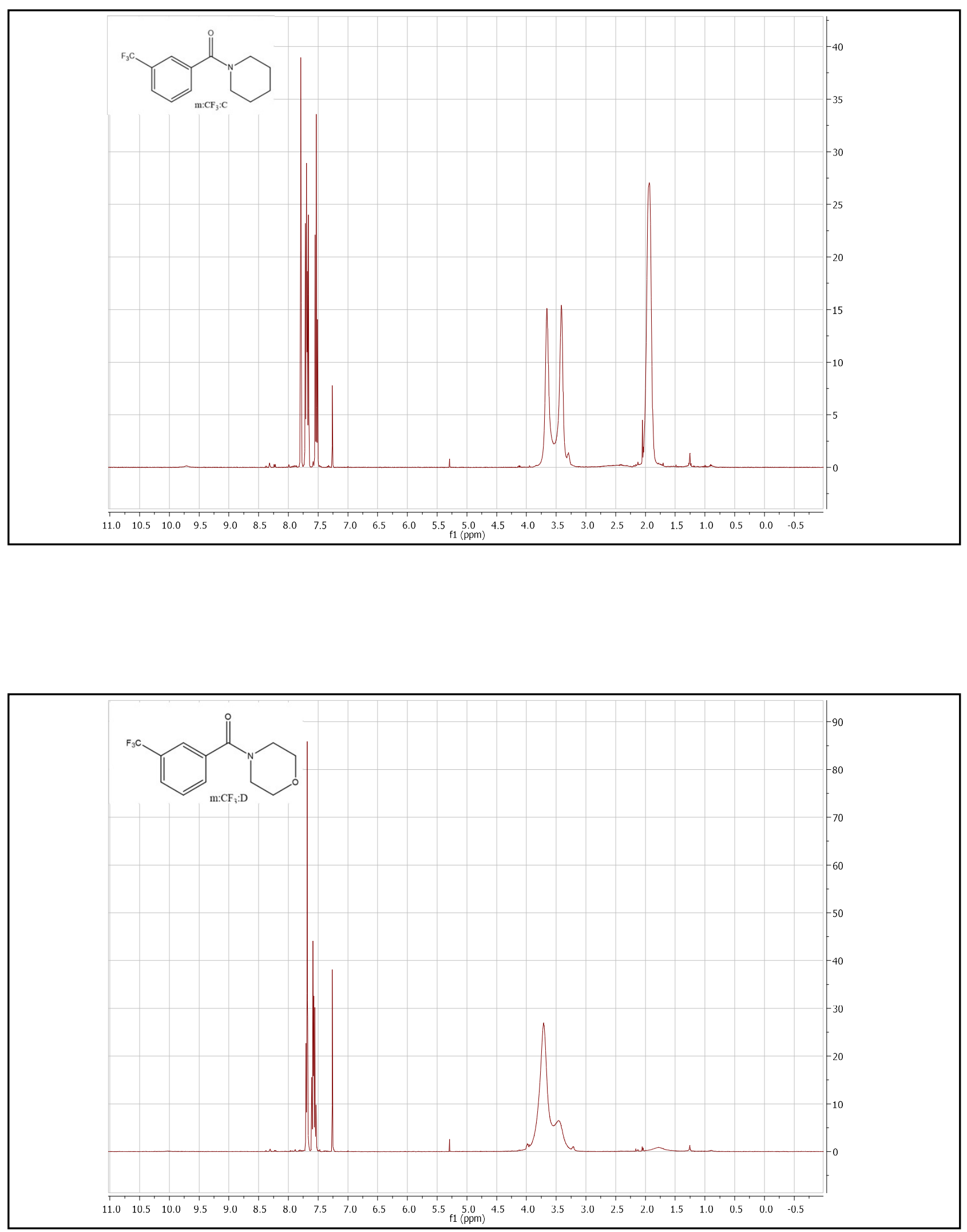

15 
Figure S2

S23
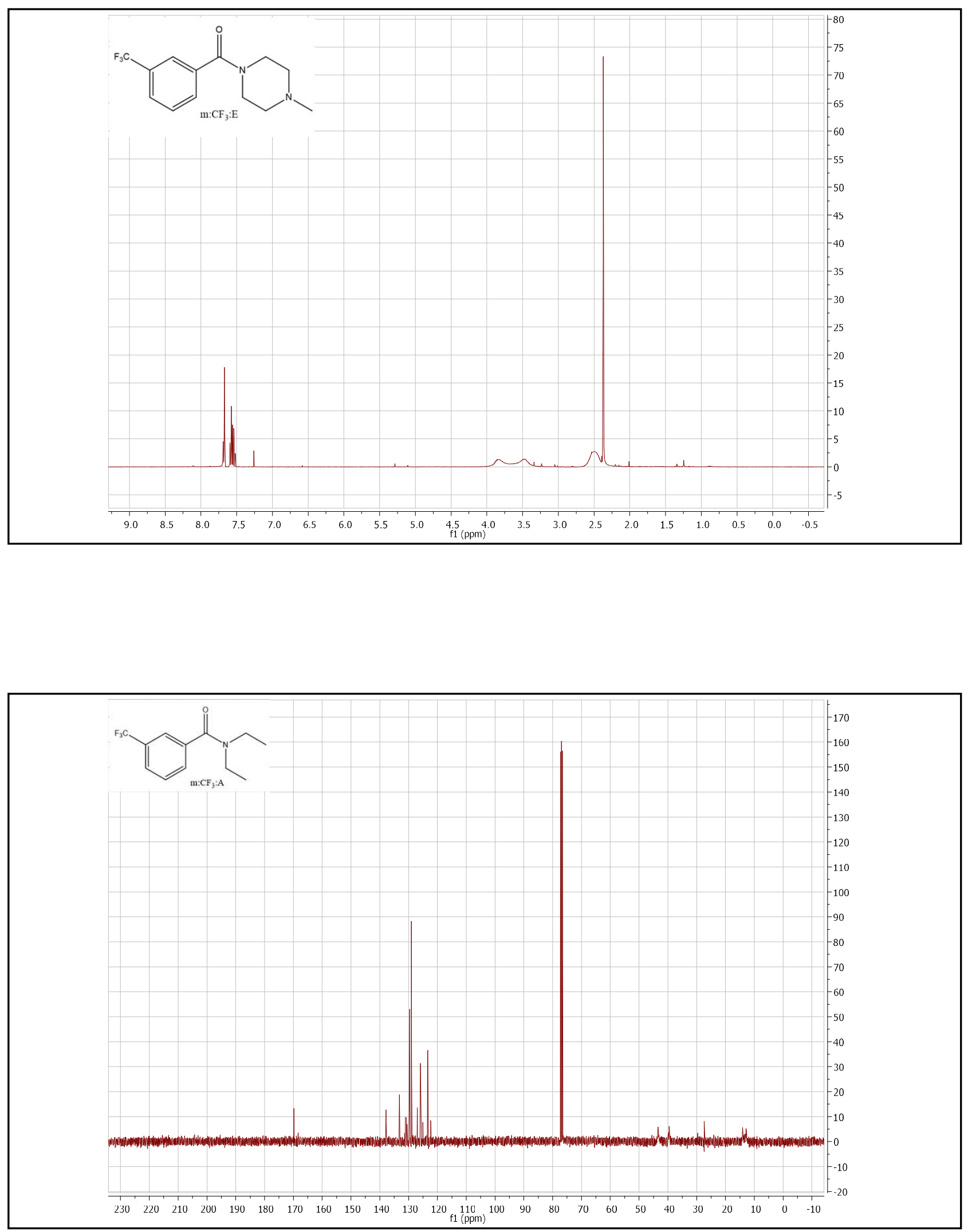

16 

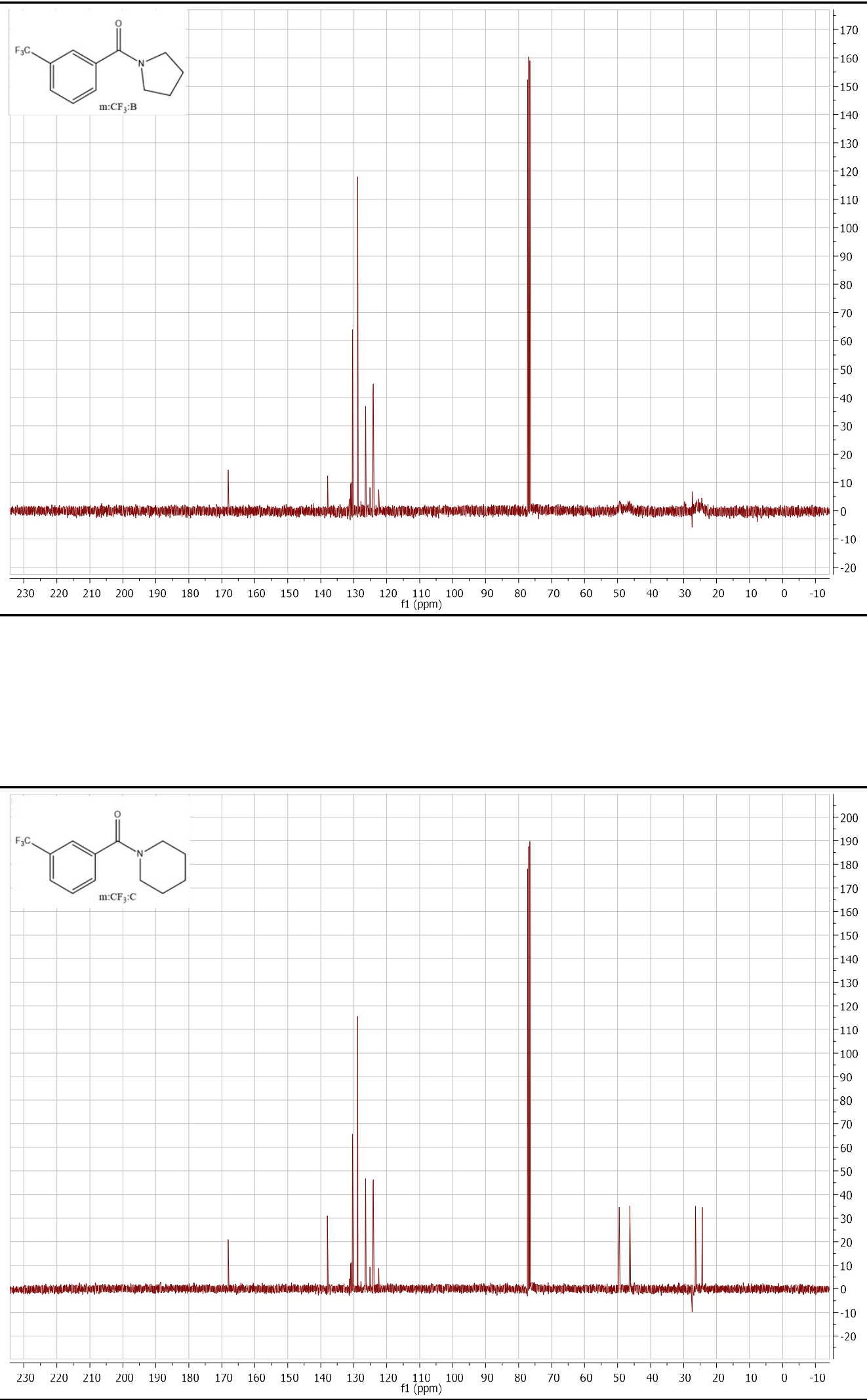
Figure S2

S25
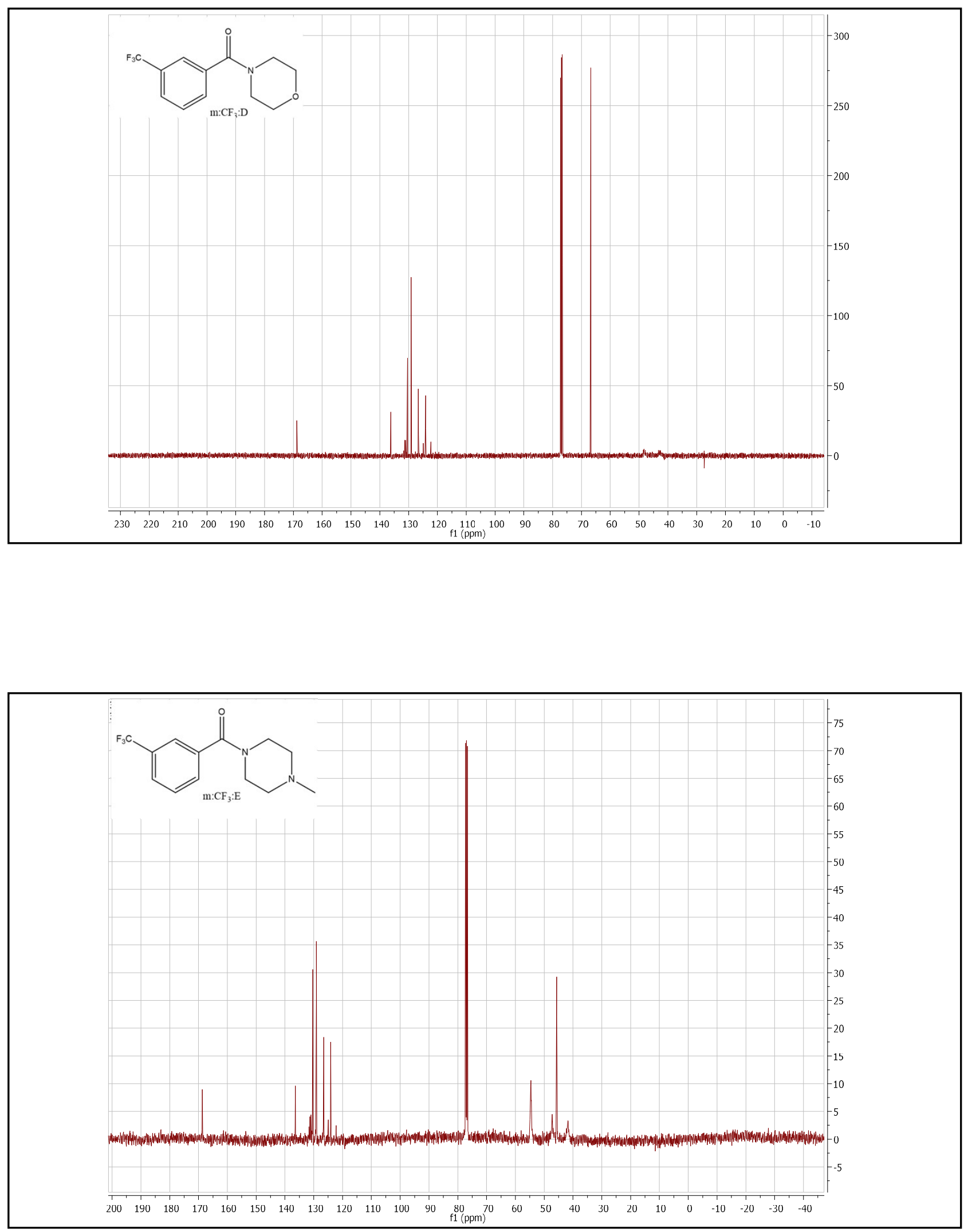

18 
Figure S2

S26
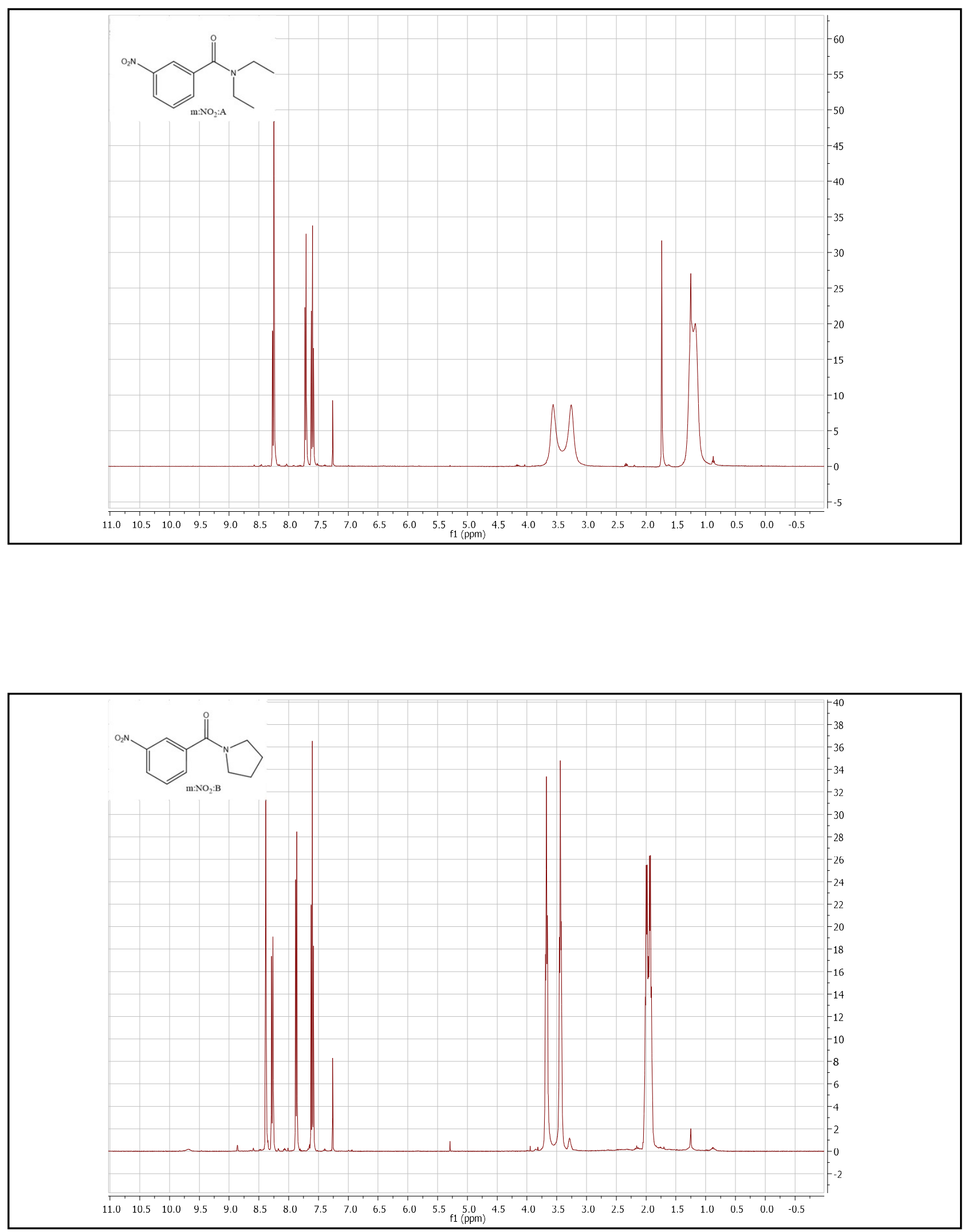

19 
Figure S2

S27
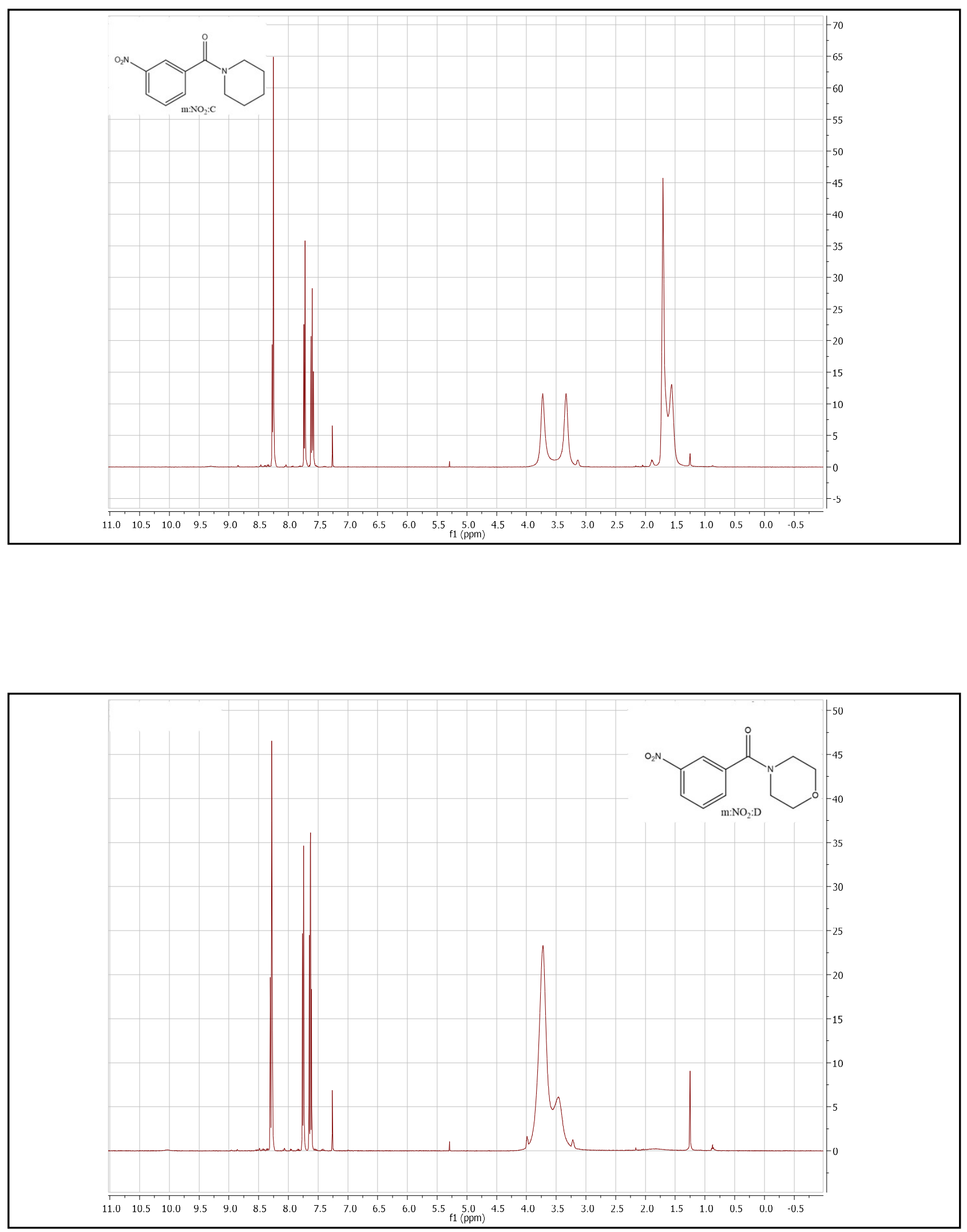

20 
Figure S2

S28
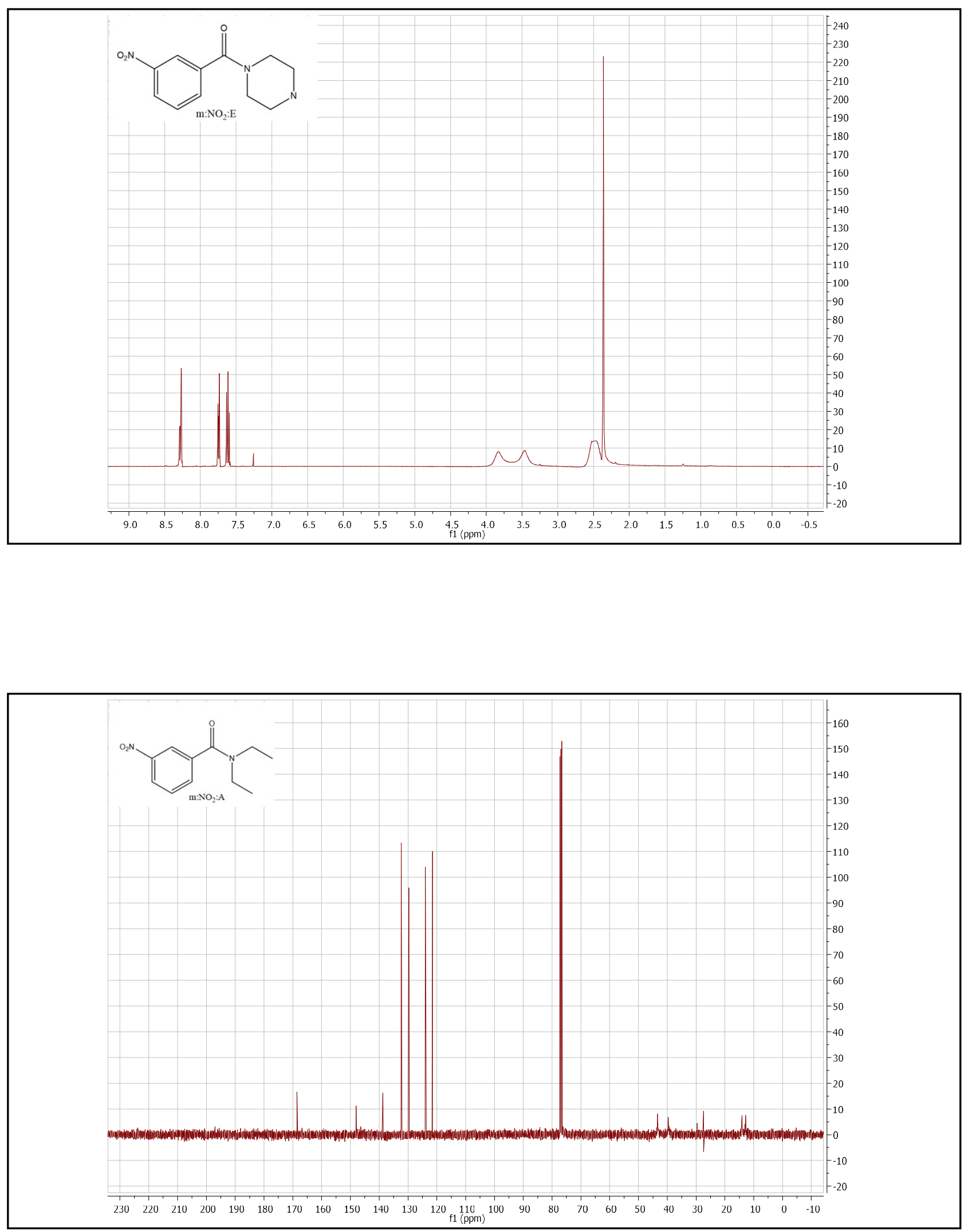

21 
Figure S2

S29
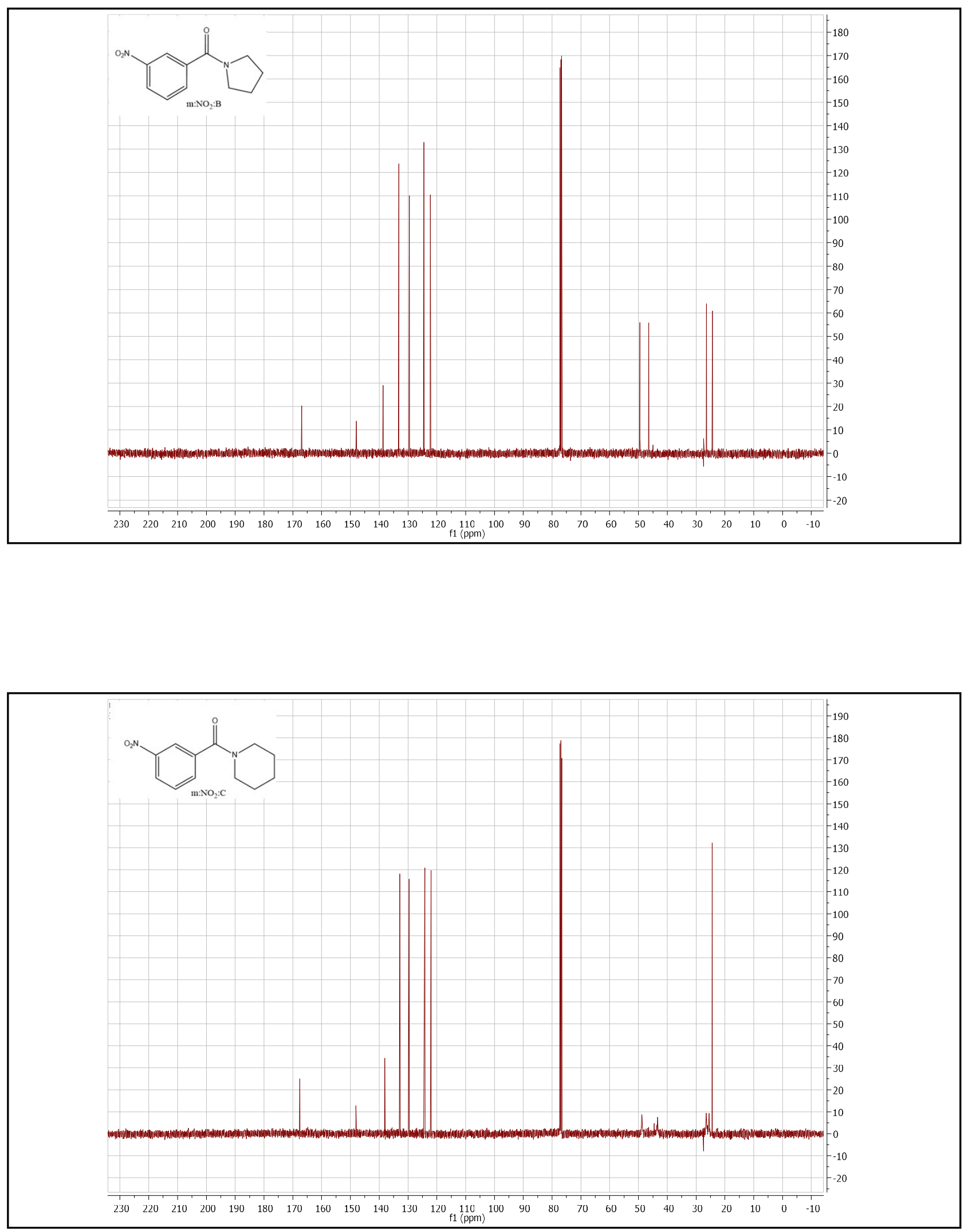

22 
Figure S2

S30
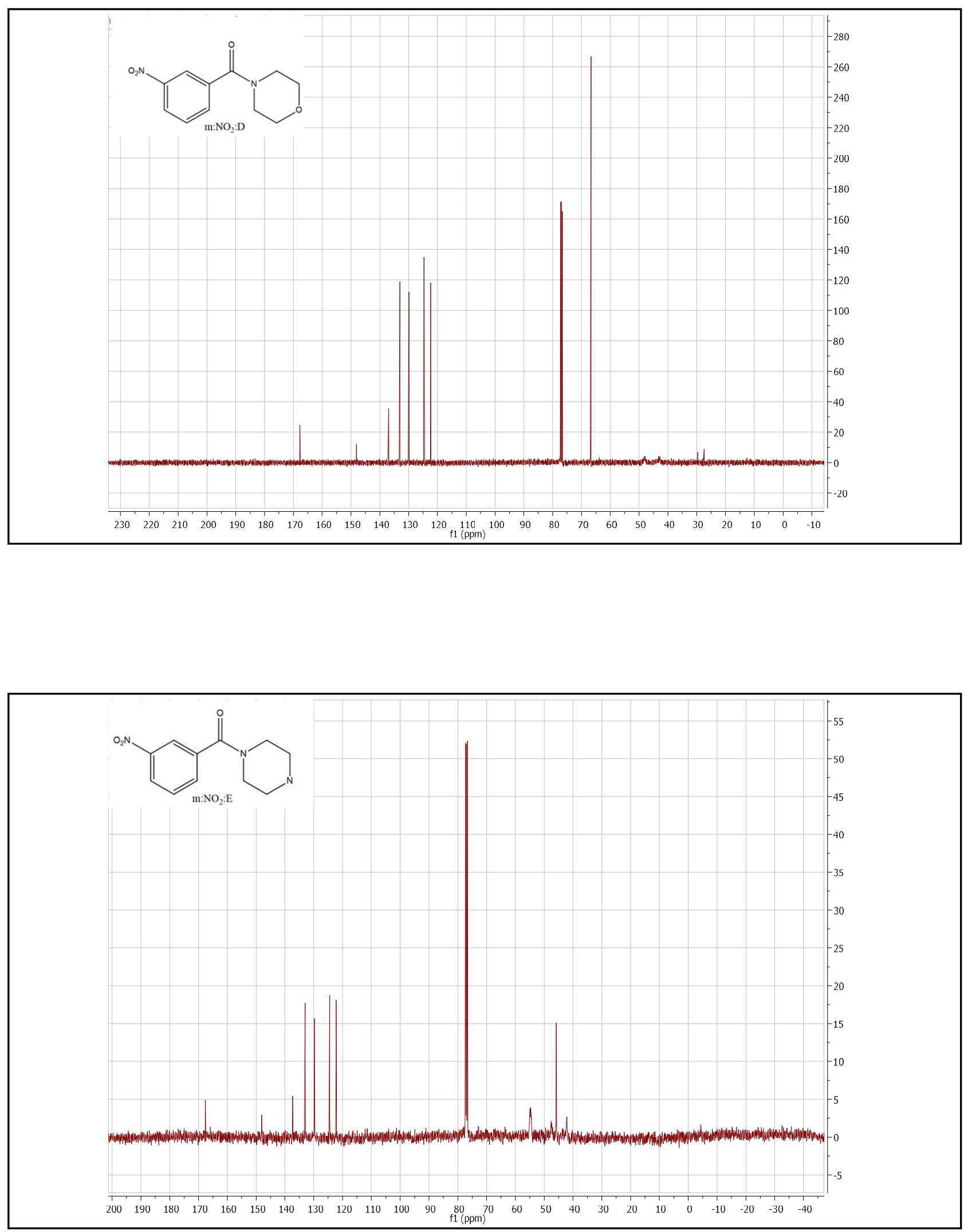

23 
Figure S2

S31
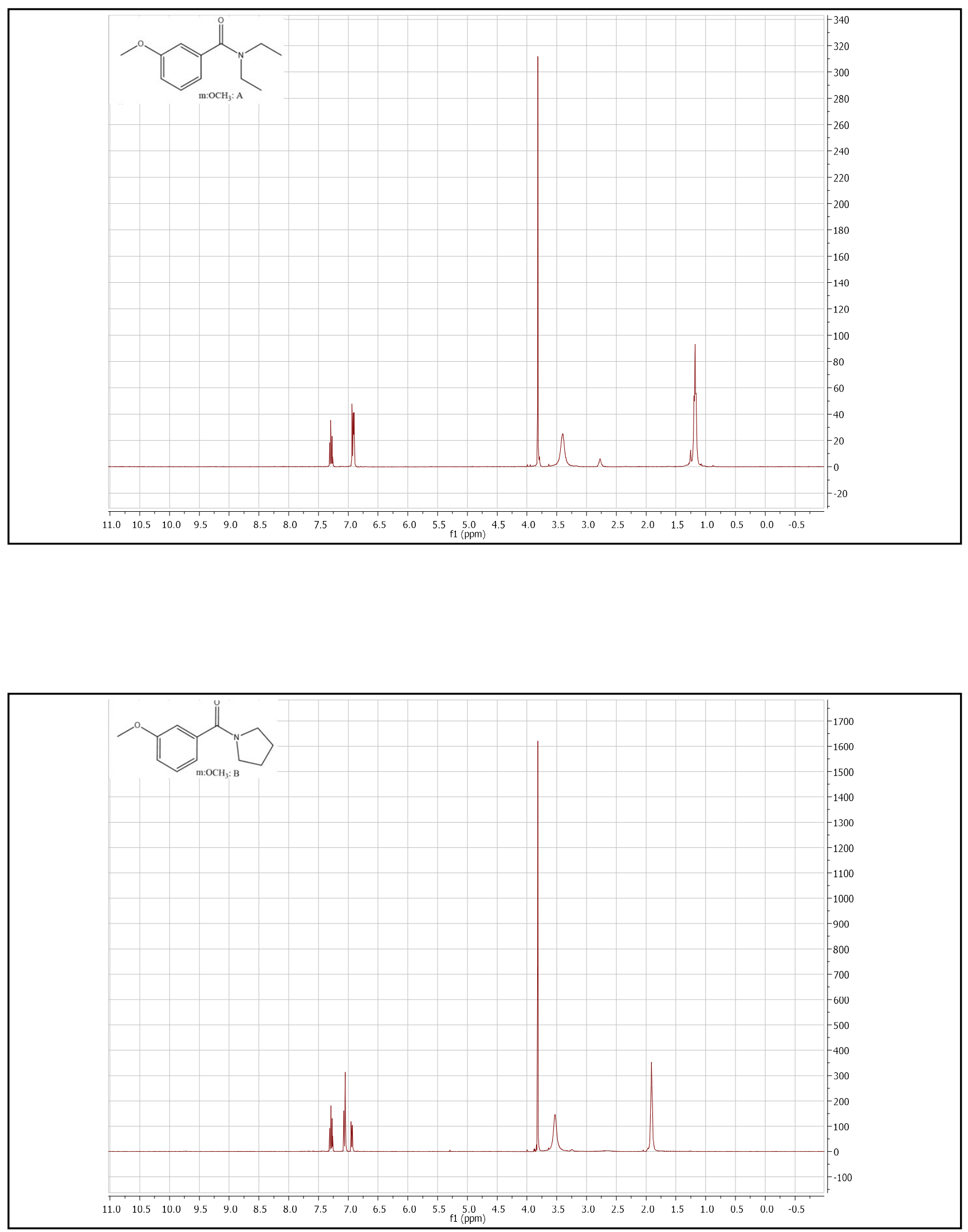

24 
Figure S2

S32
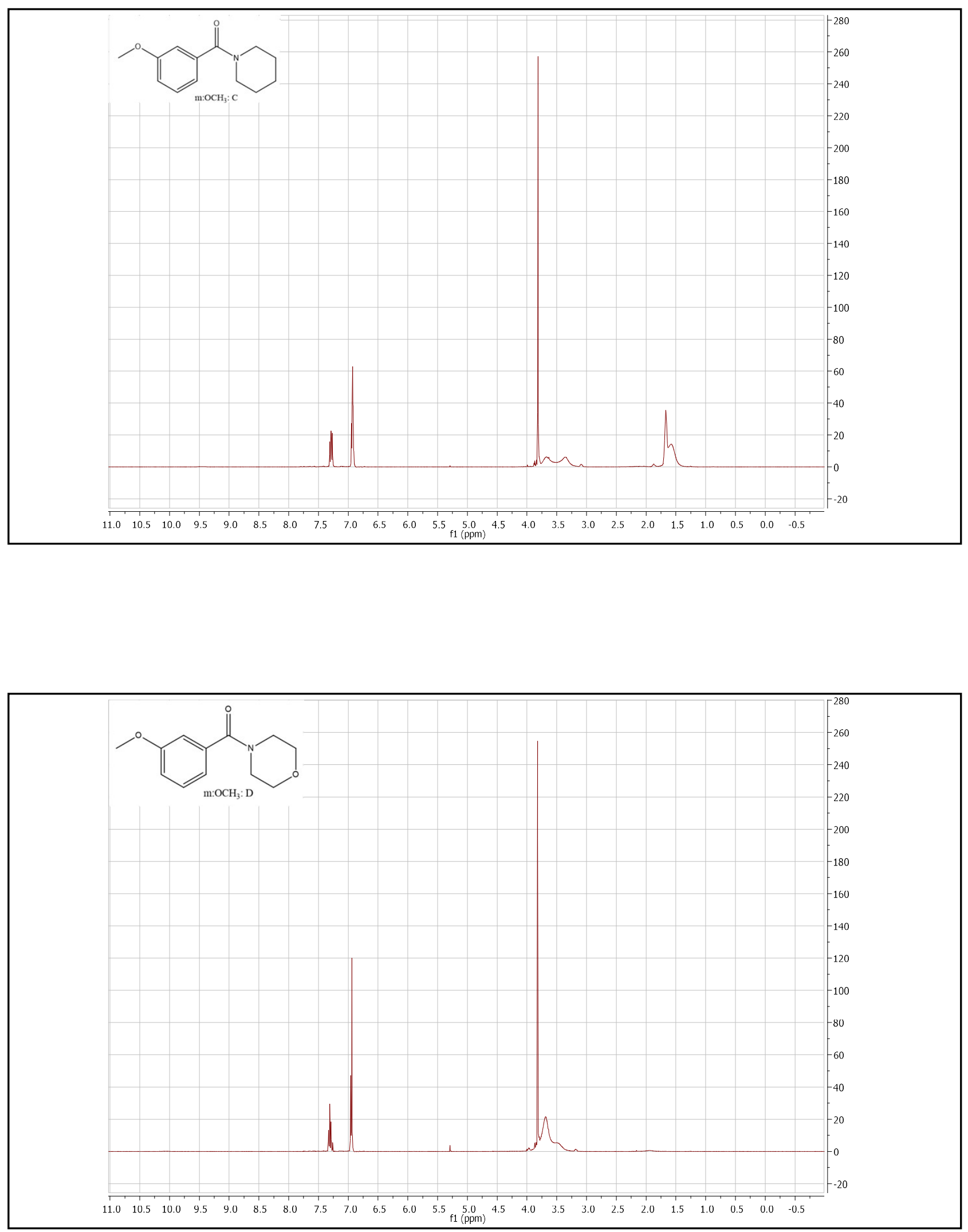

25 
Figure S2

S33
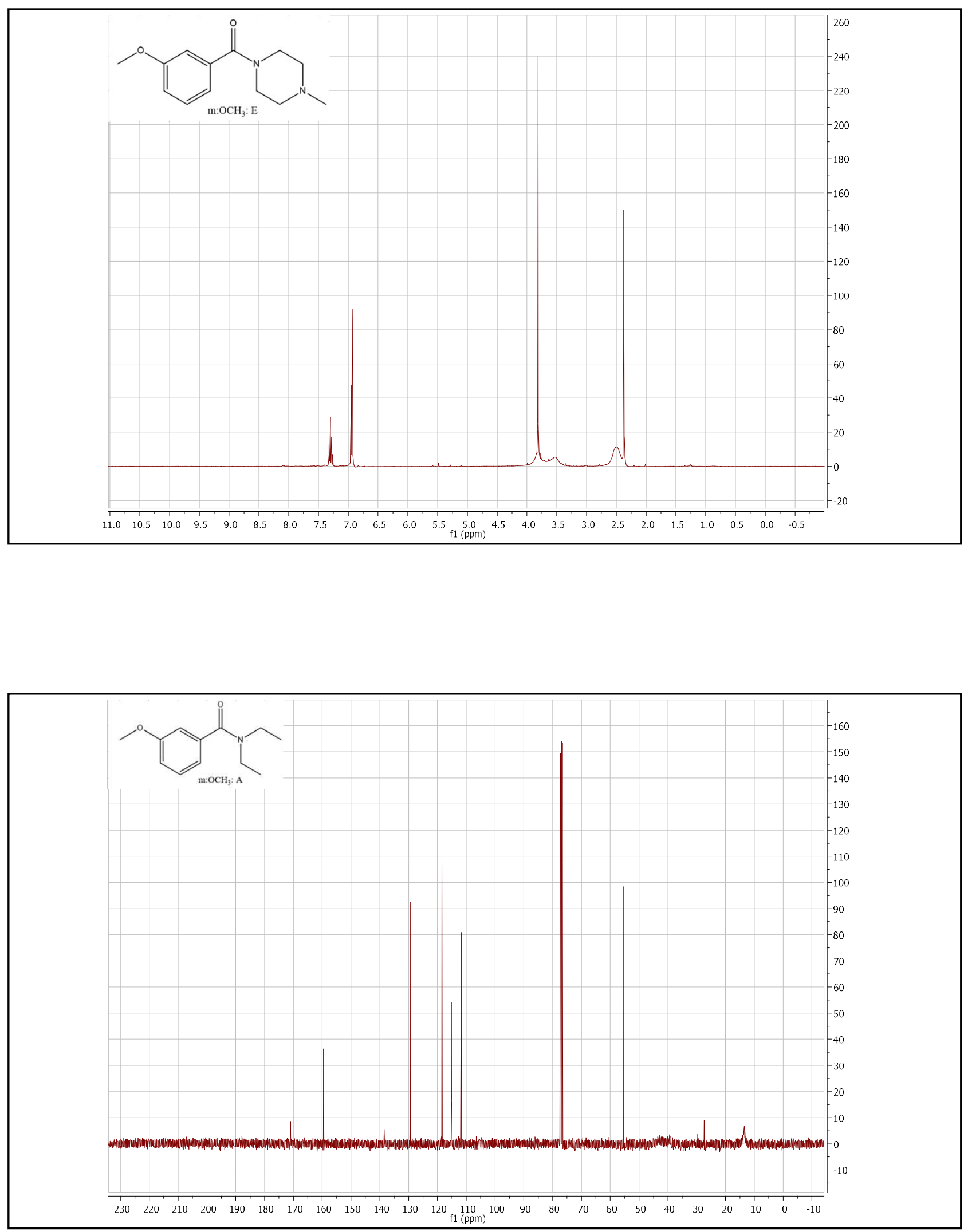

26 
Figure S2

S34
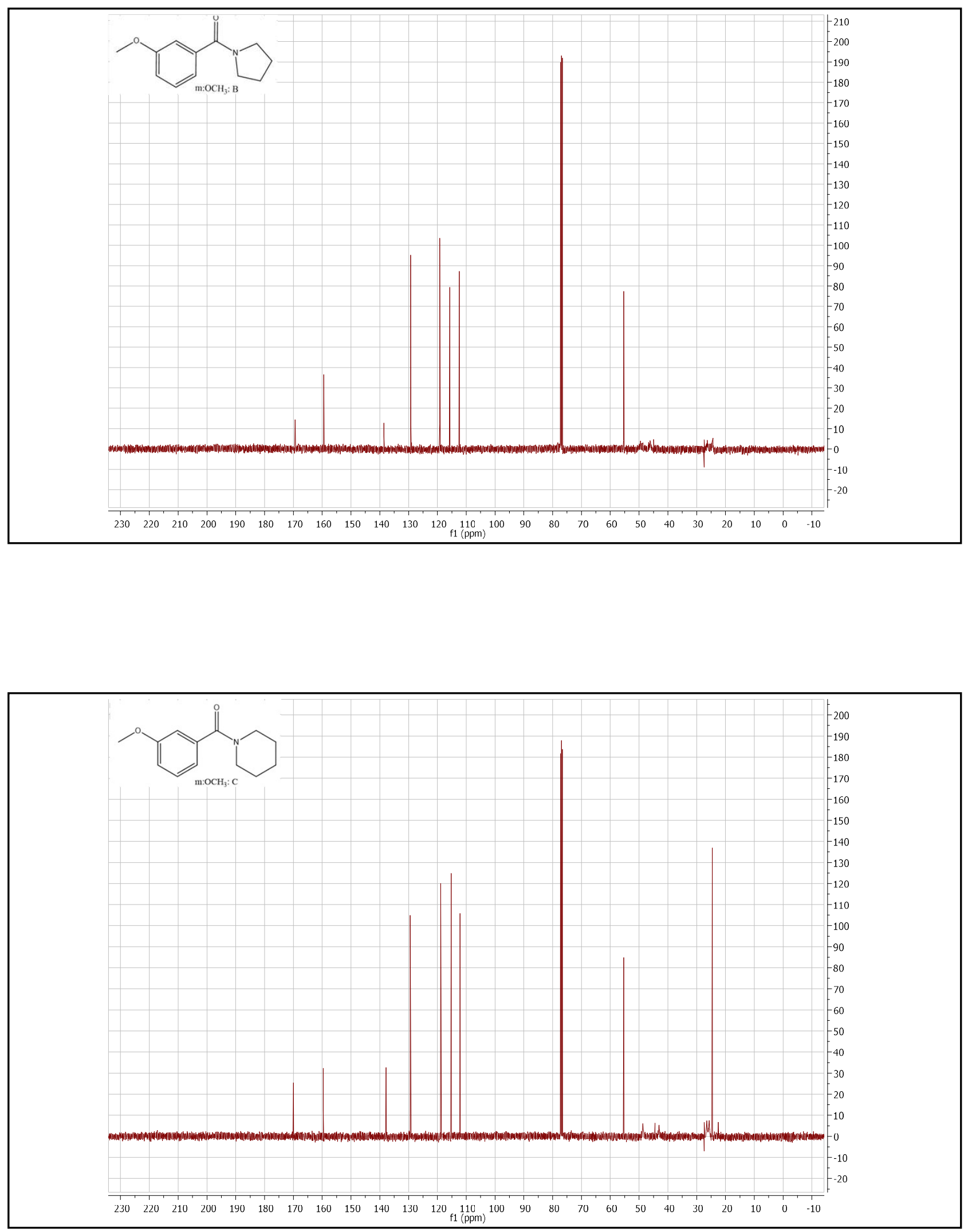

27 
Figure S2

S35
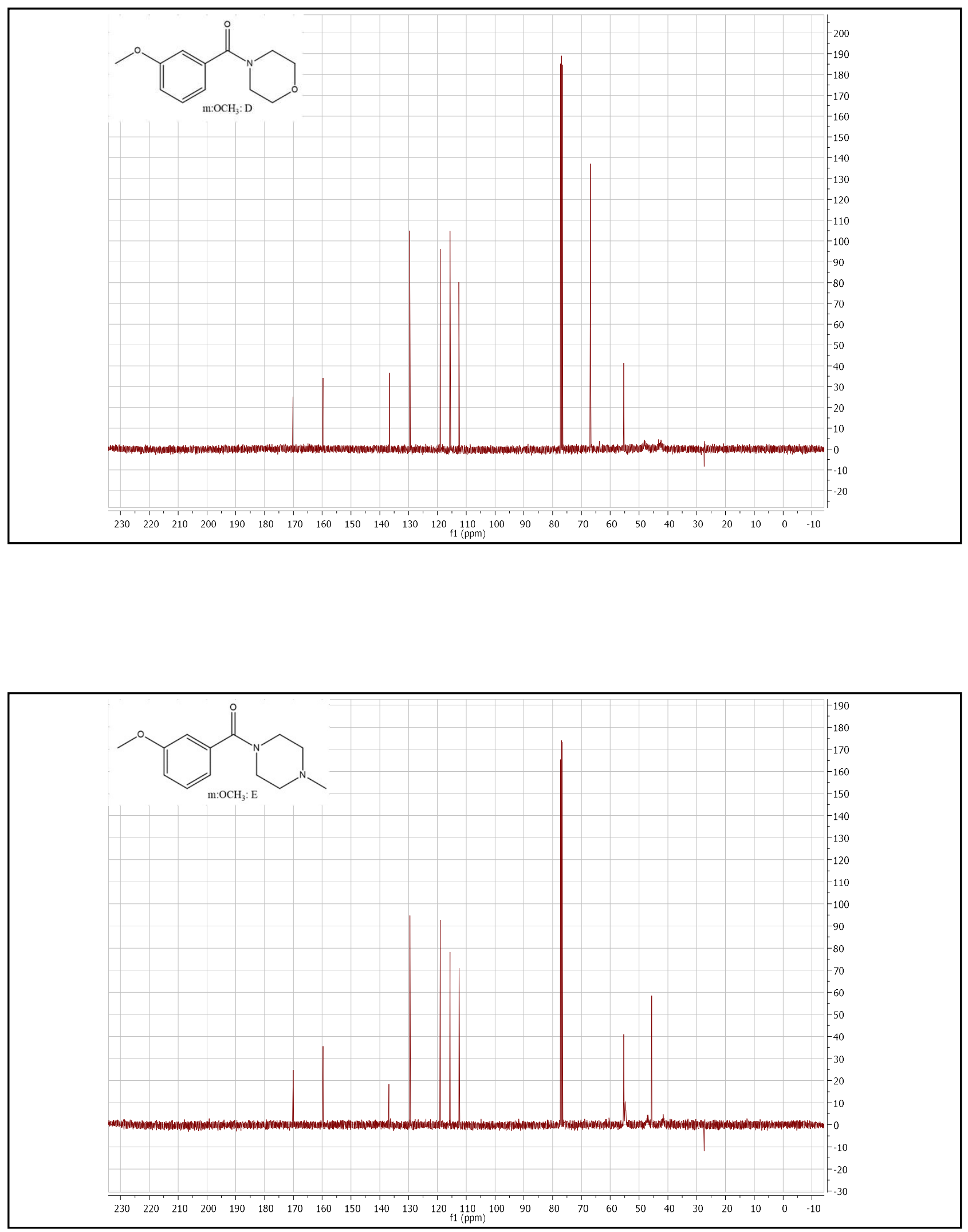

28 
Figure S2

S36
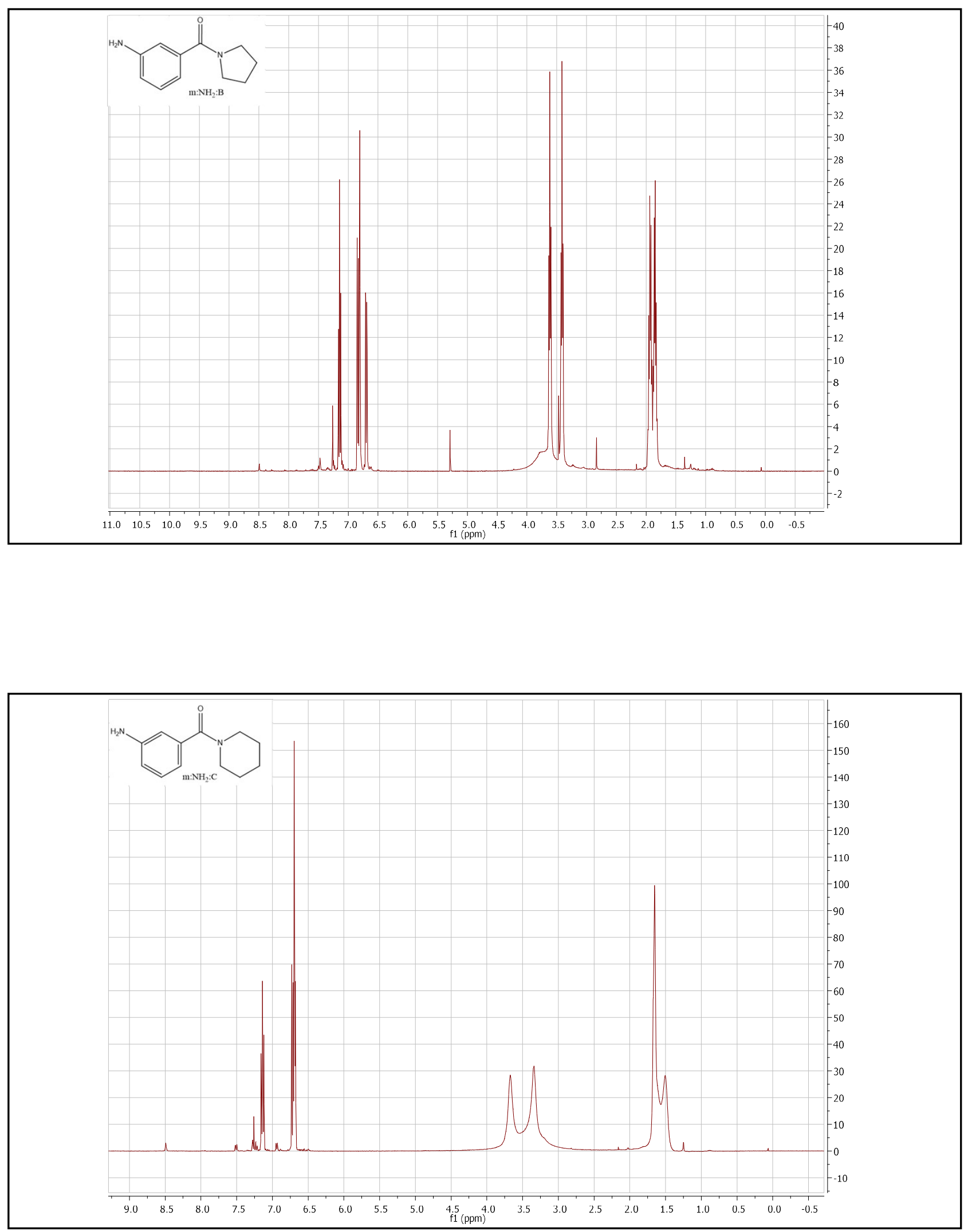

29 
Figure S2

S37
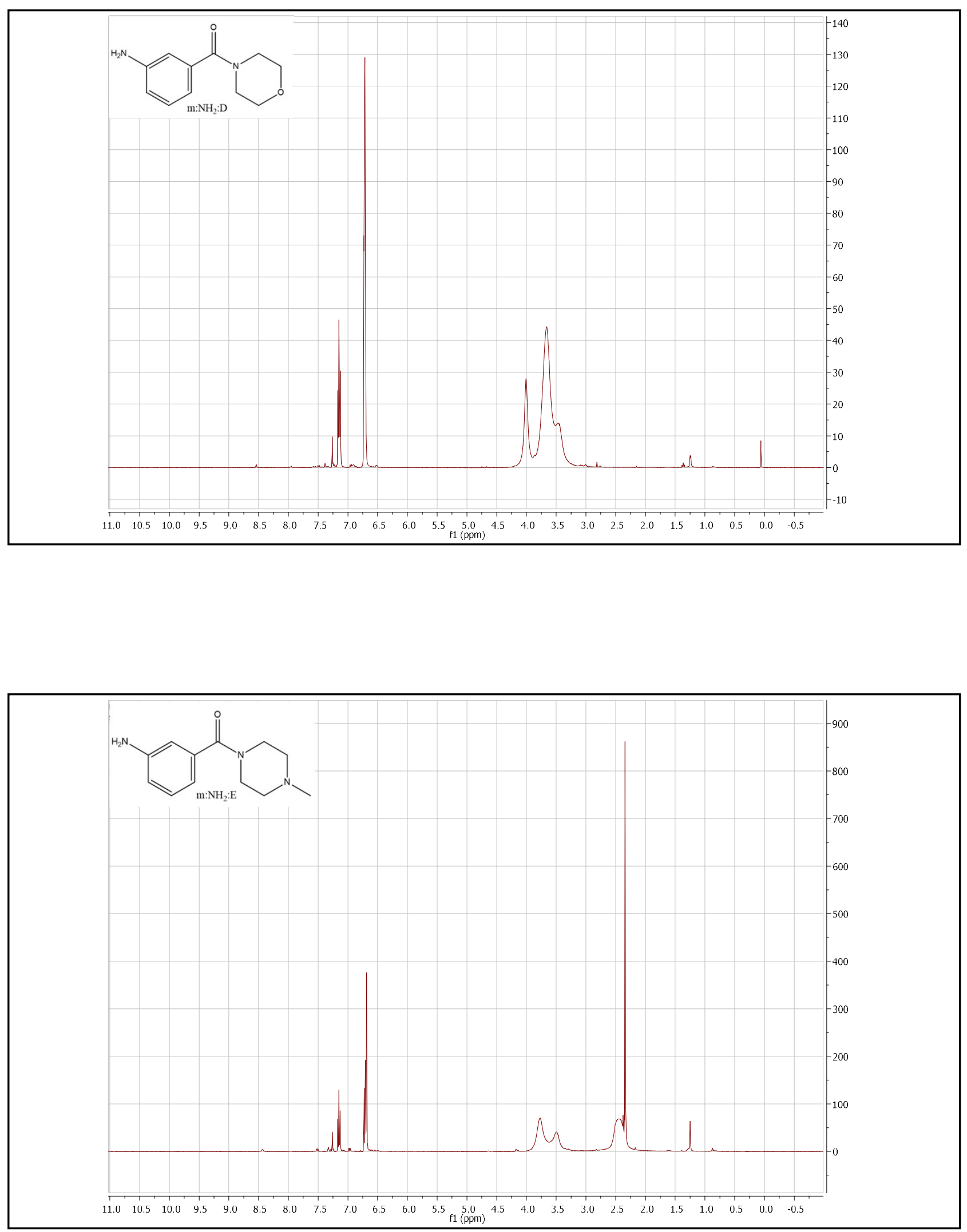

30 
Figure S2

S38
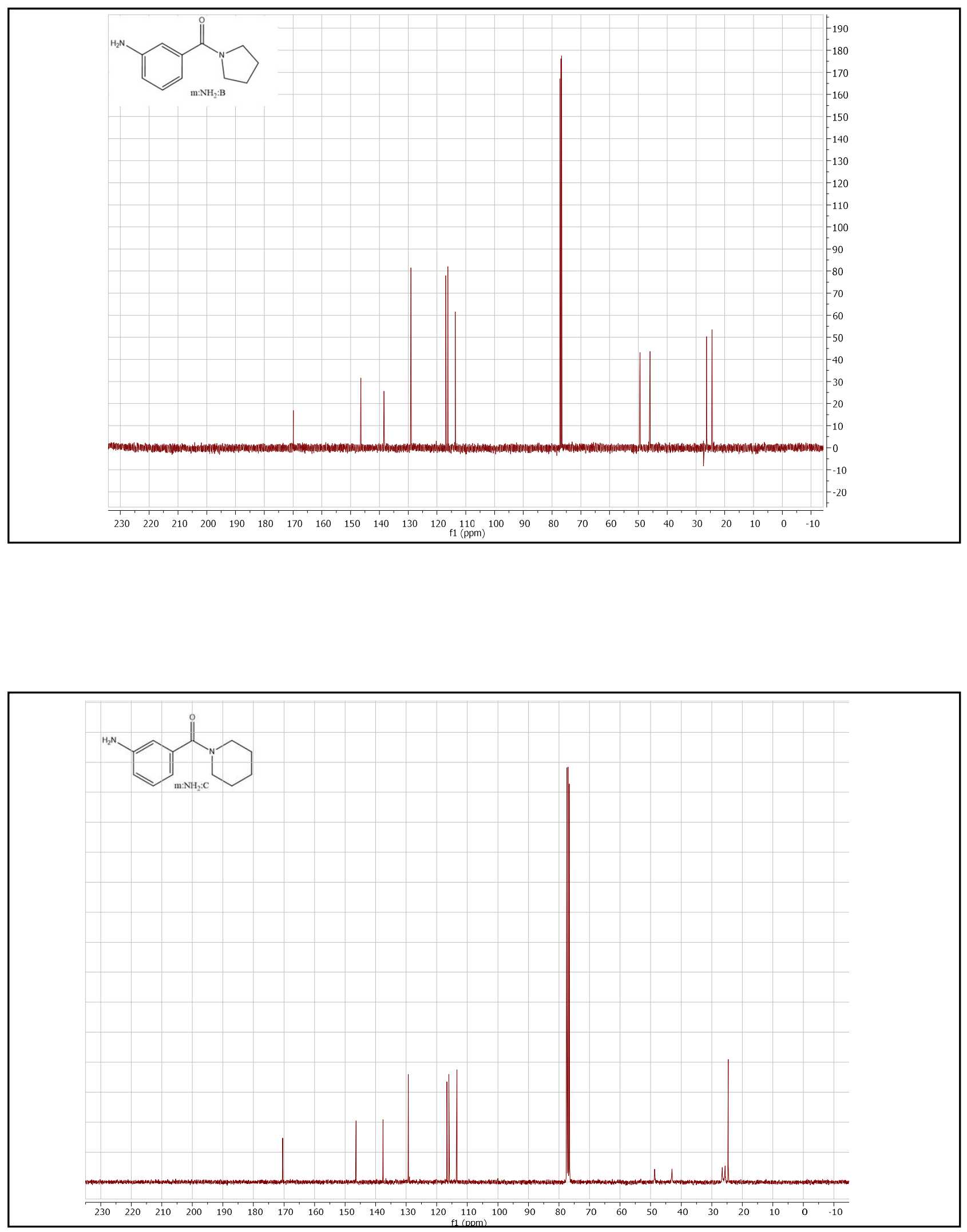

31 
Figure S2

S39
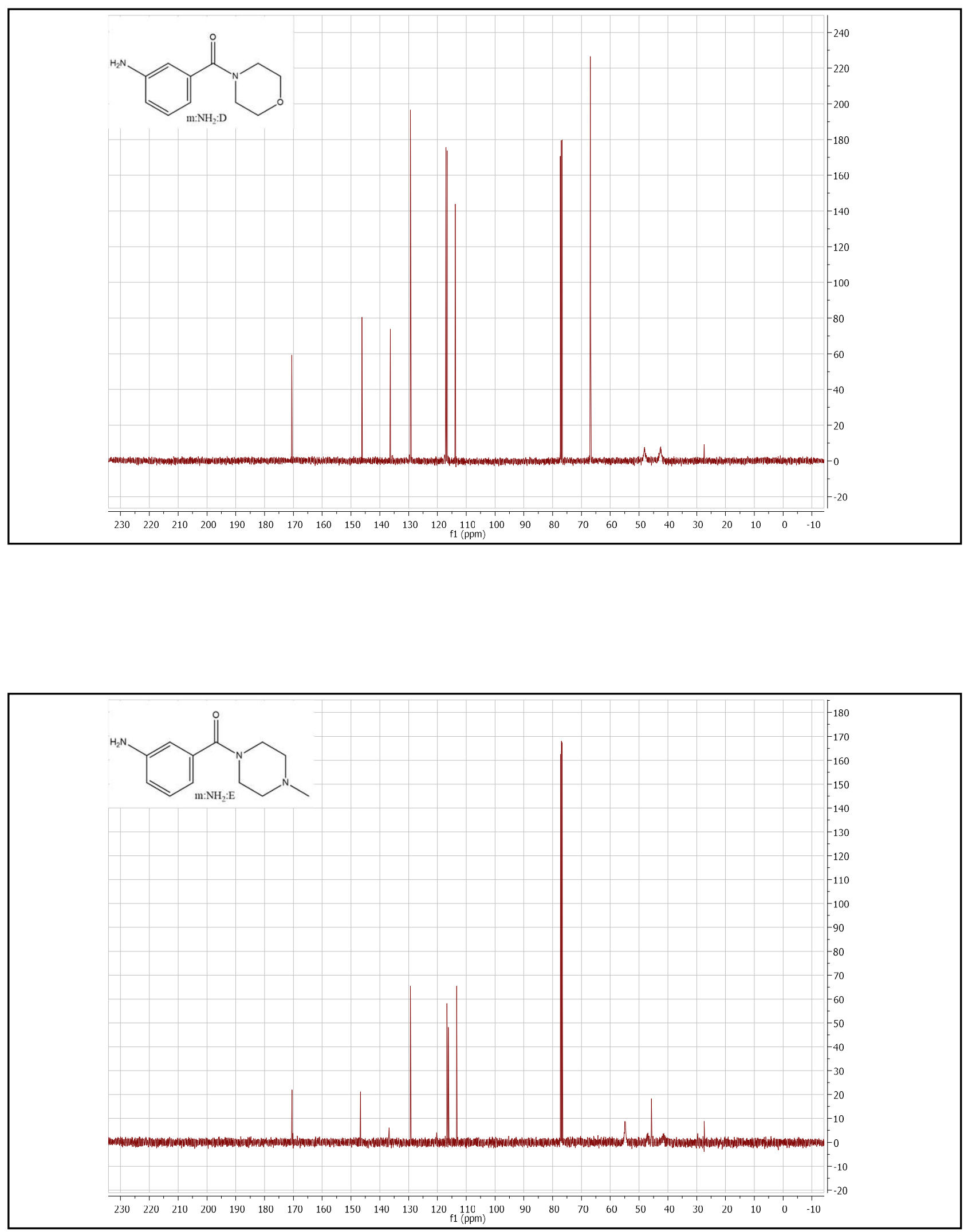

32 
Figure S2

S40
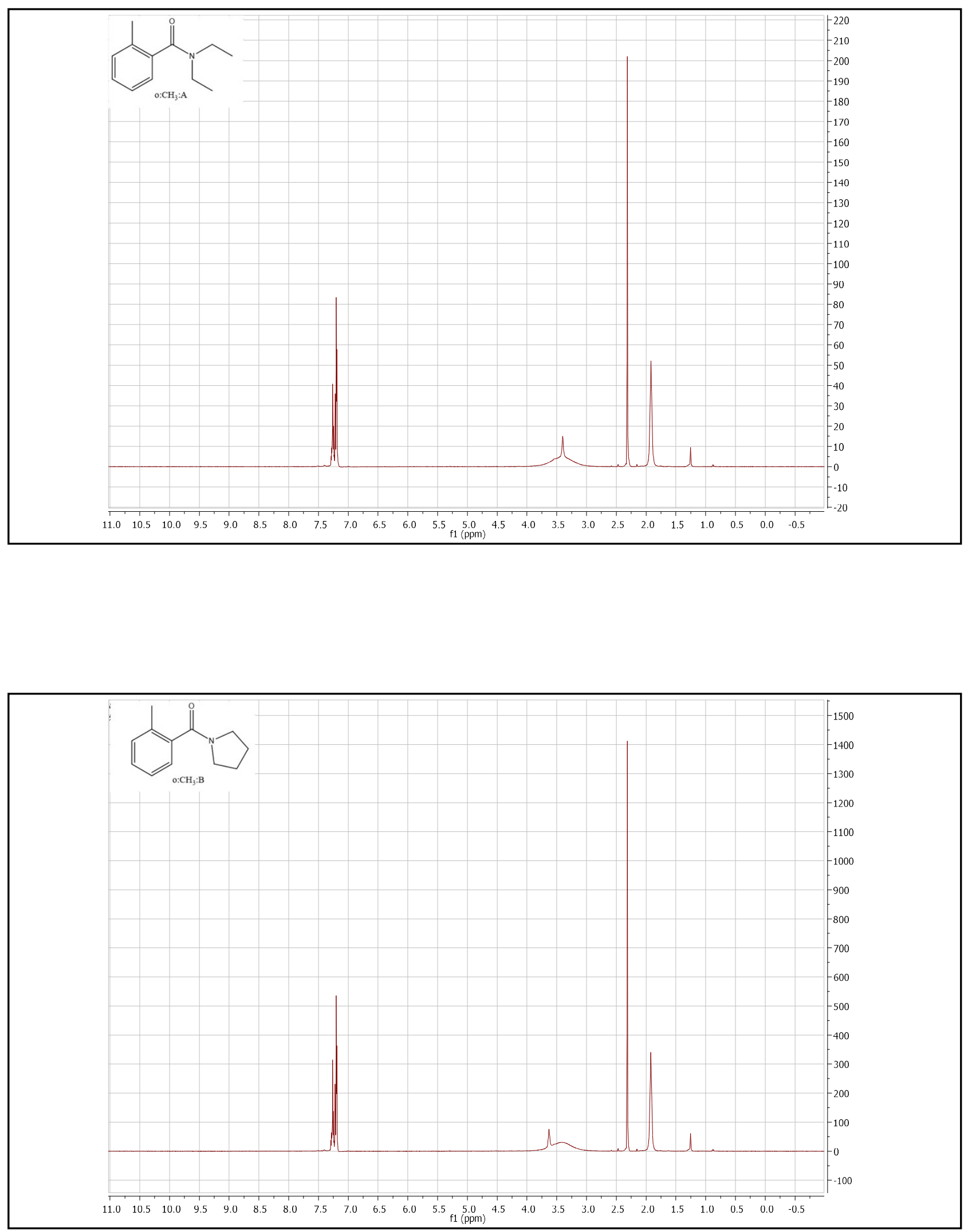

33 
Figure S2

S41
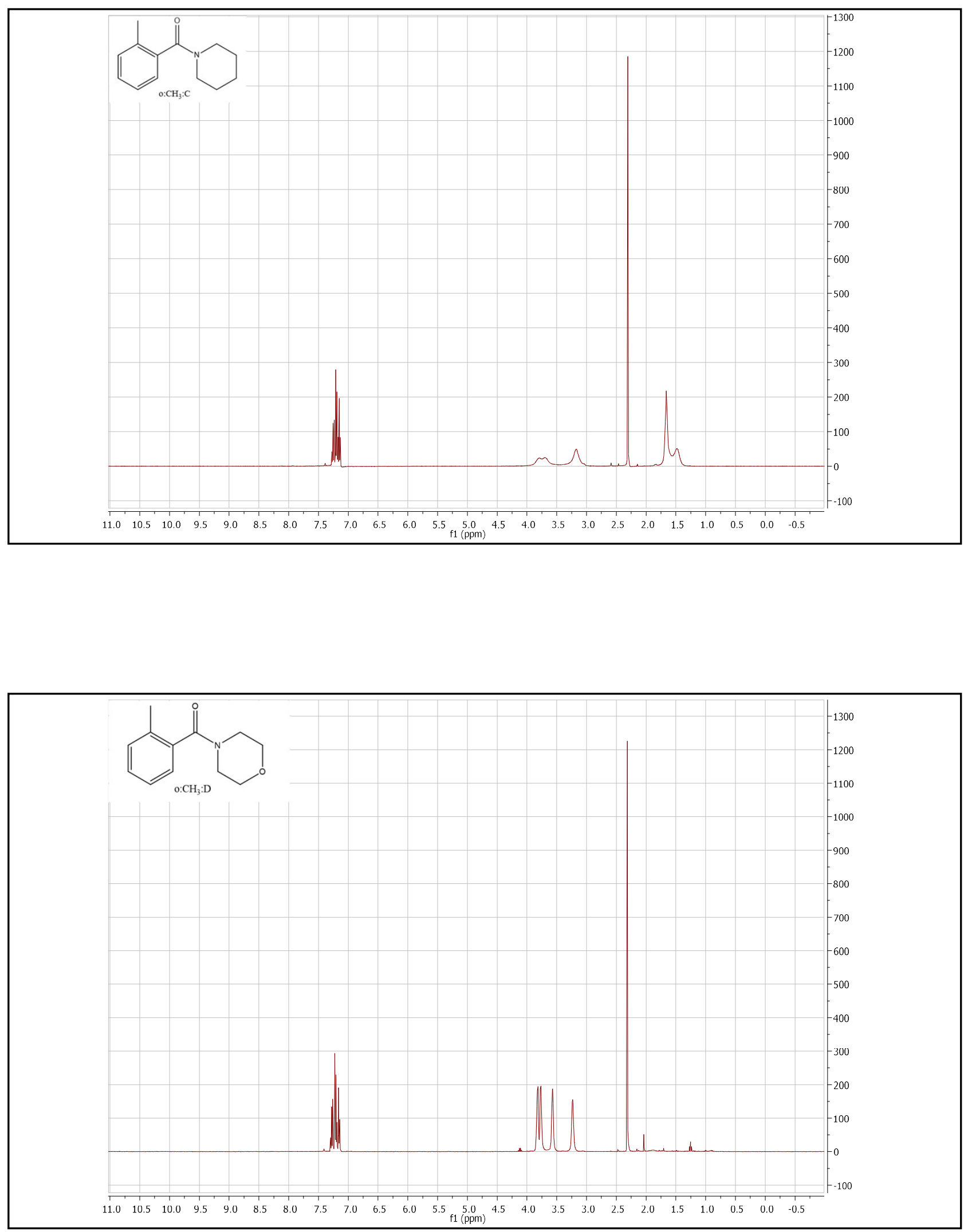

34 
Figure S2

S42
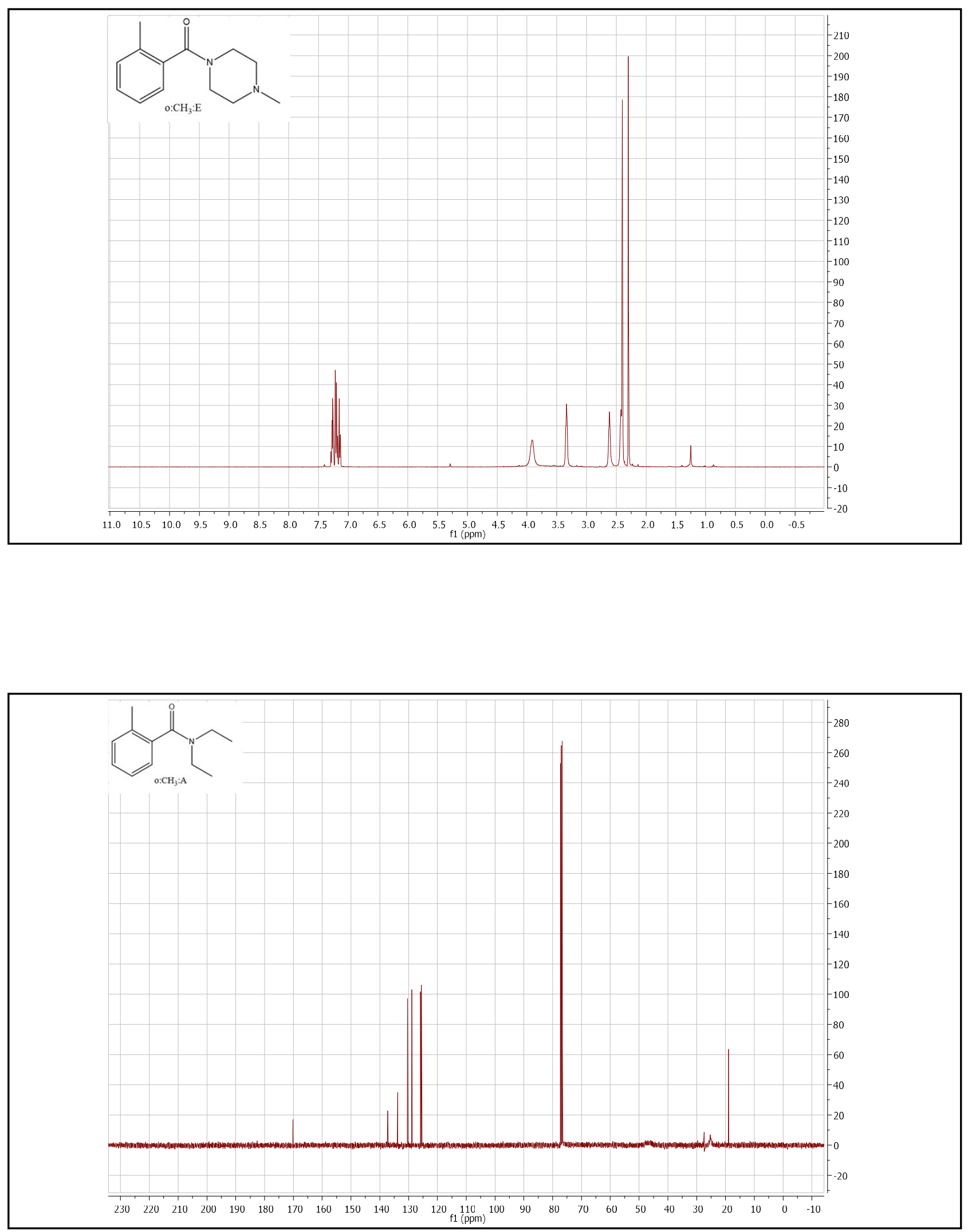

35 
Figure S2

S43
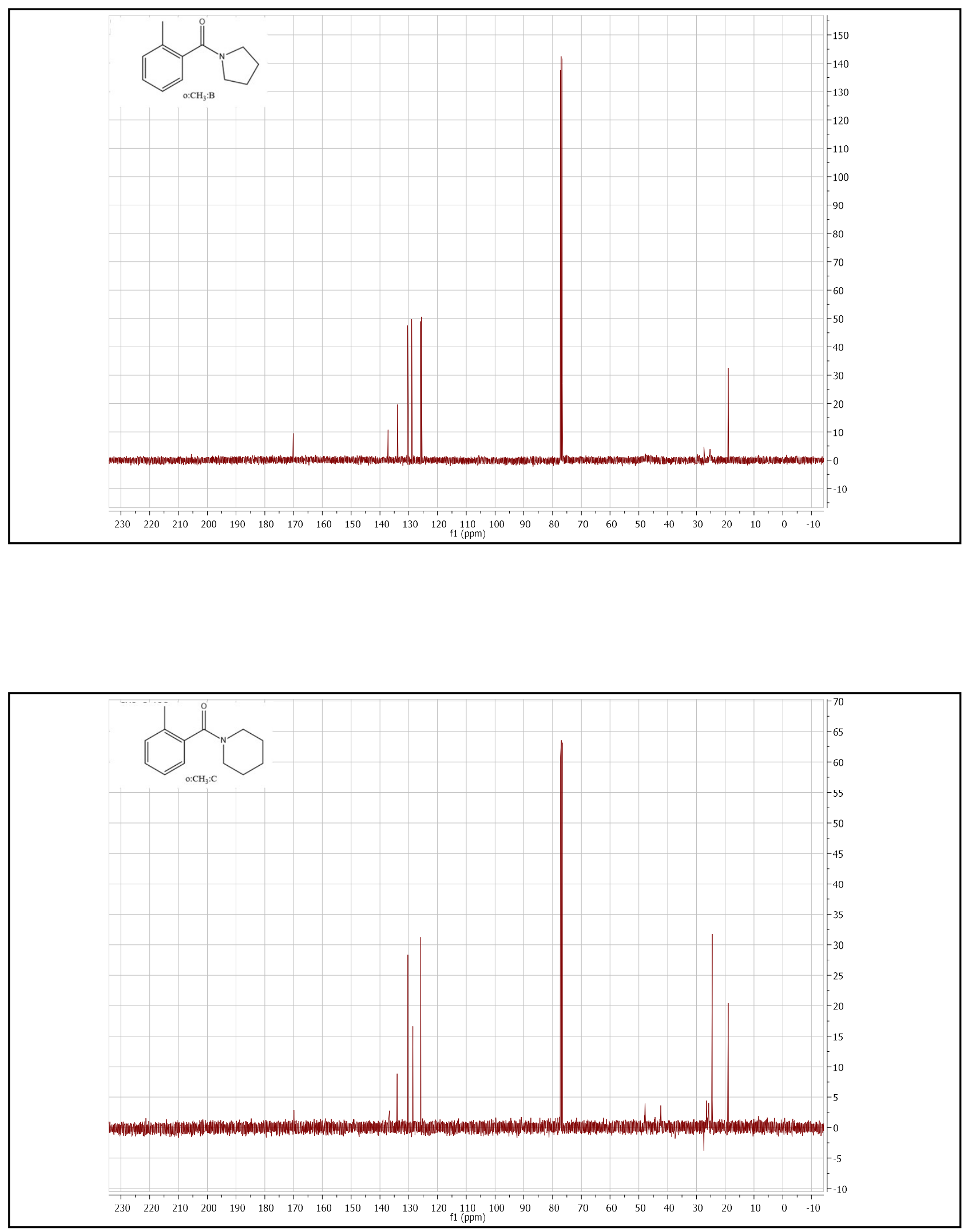

36 
Figure S2

S44
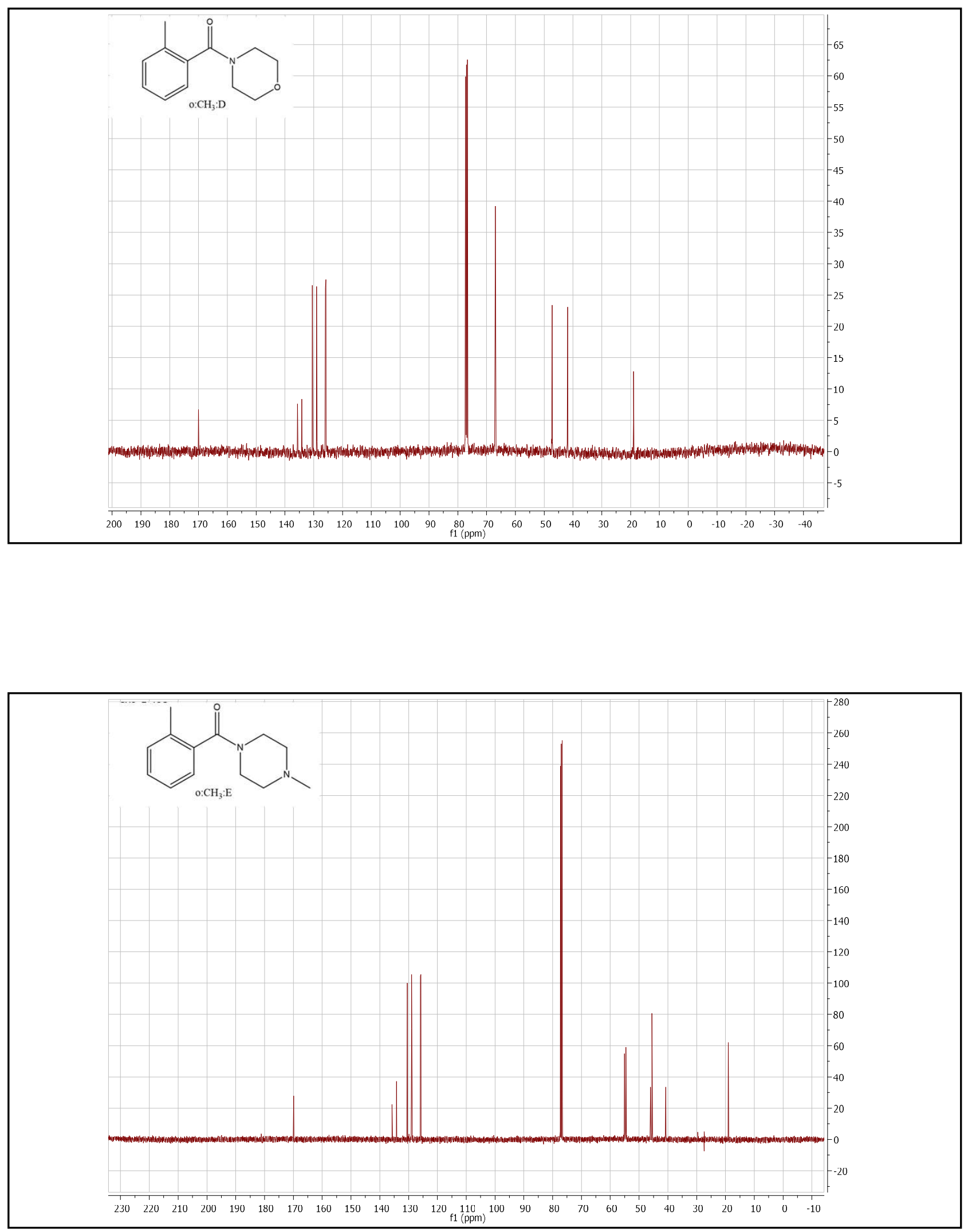

37 
Figure S2

S45
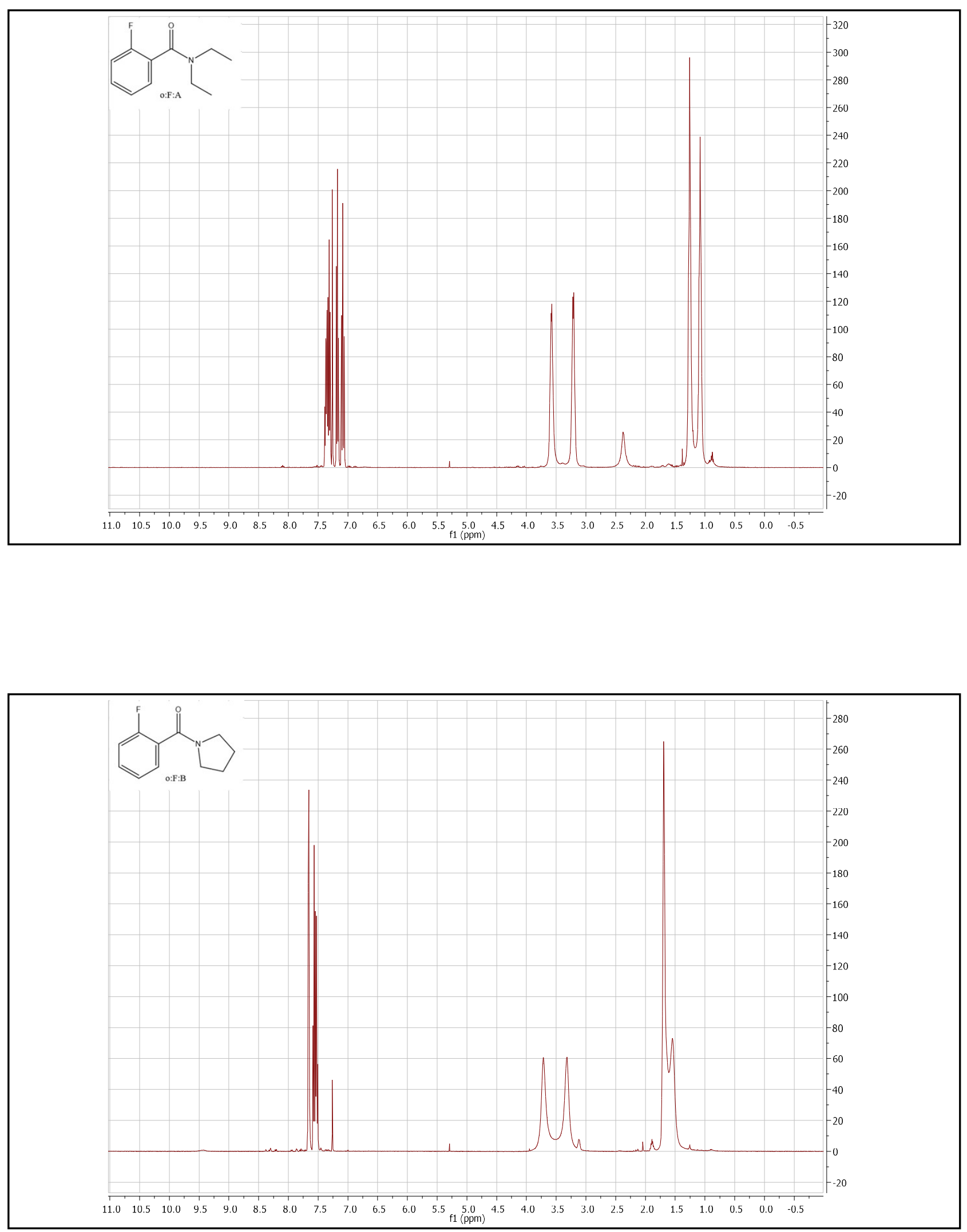

38 
Figure S2

S46
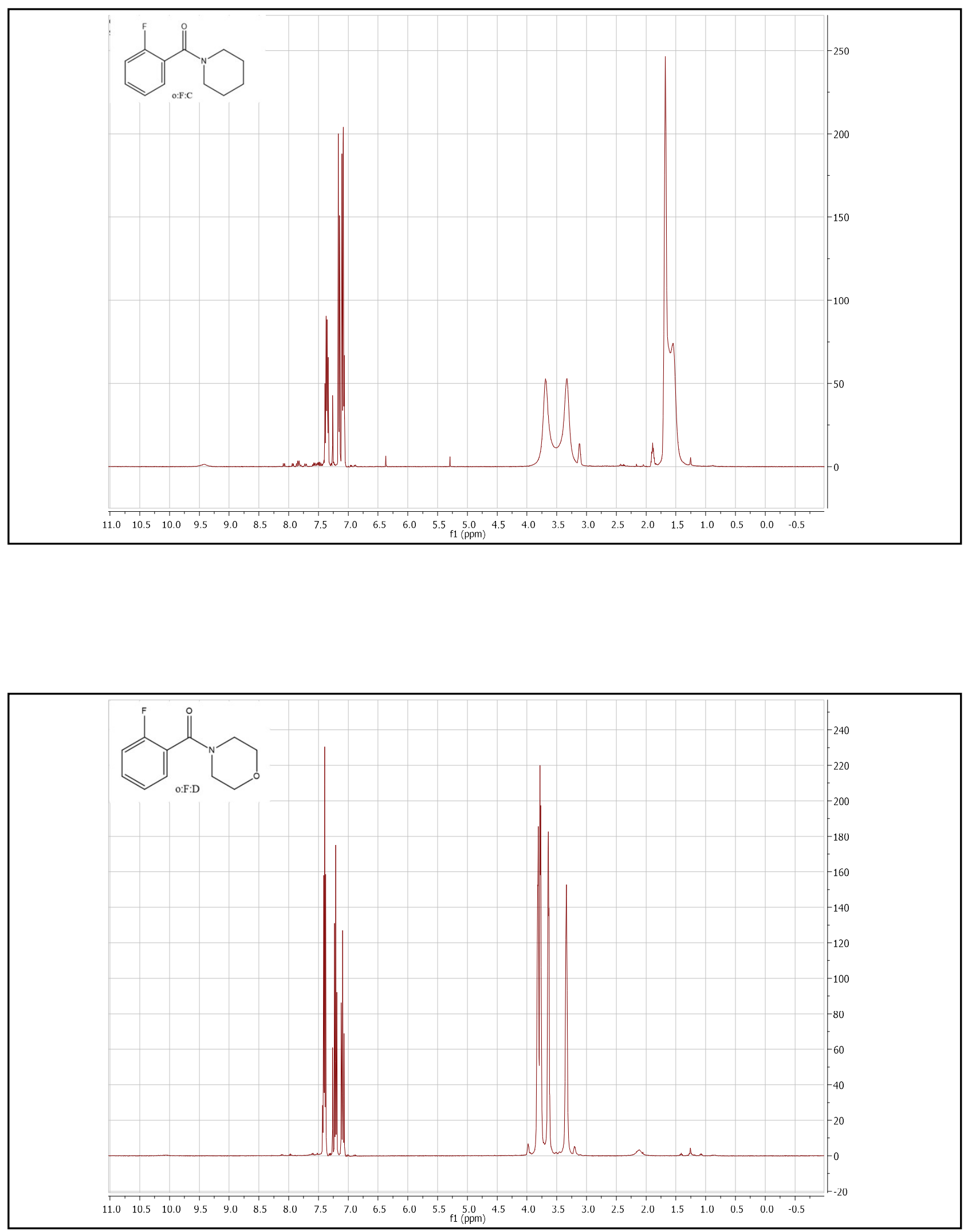

39 
Figure S2

S47
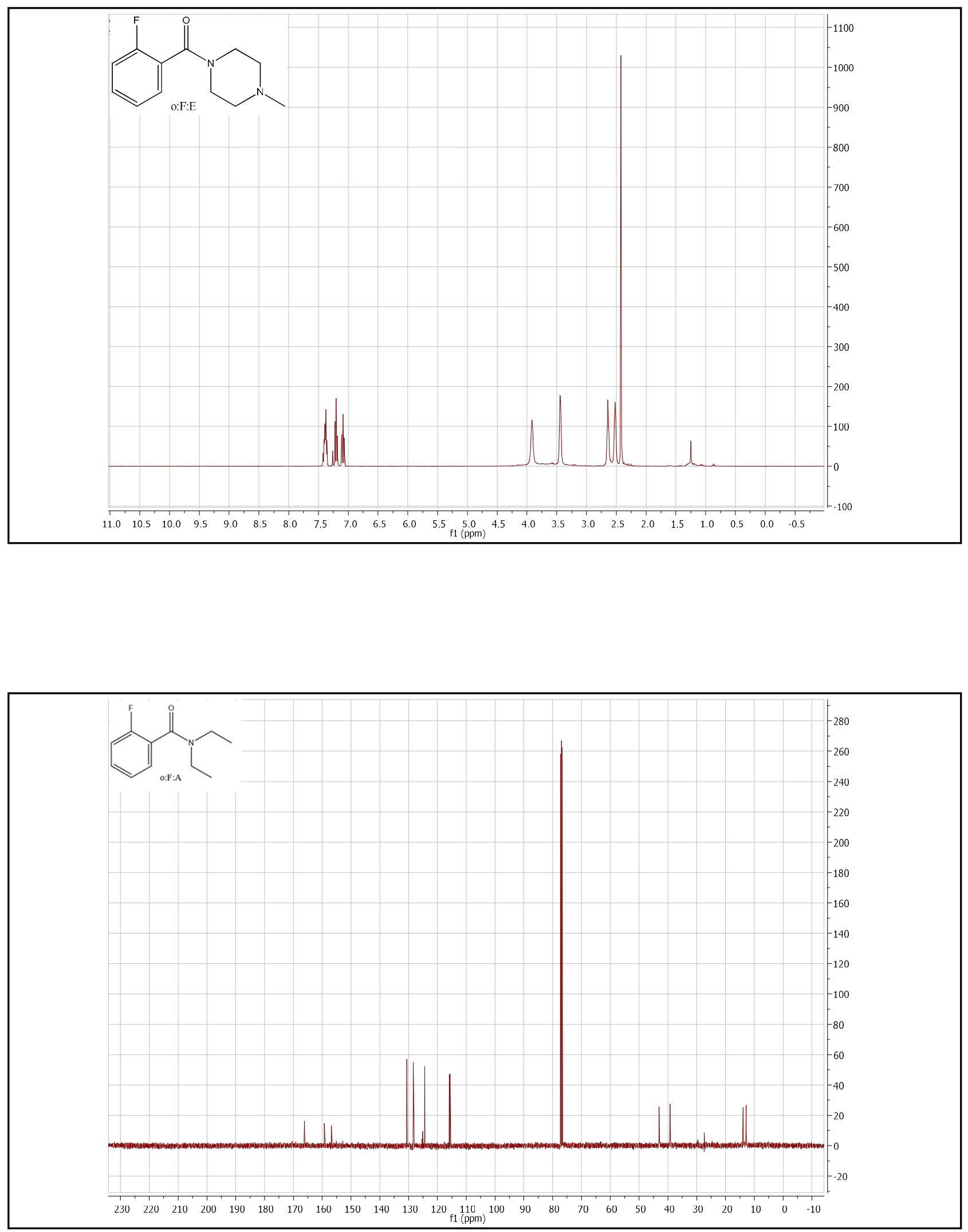

40 
Figure S2

S48
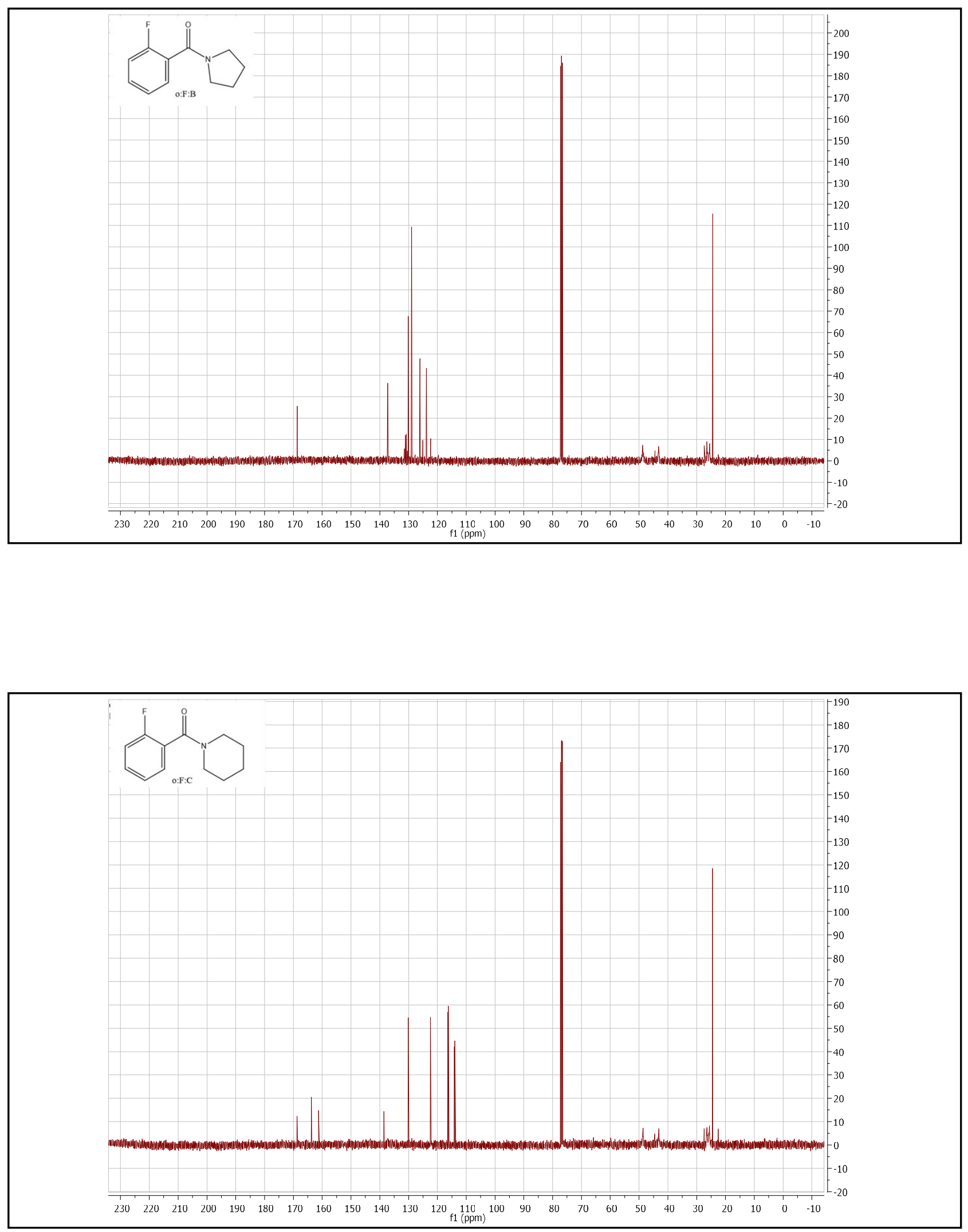

41 
Figure S2

S49
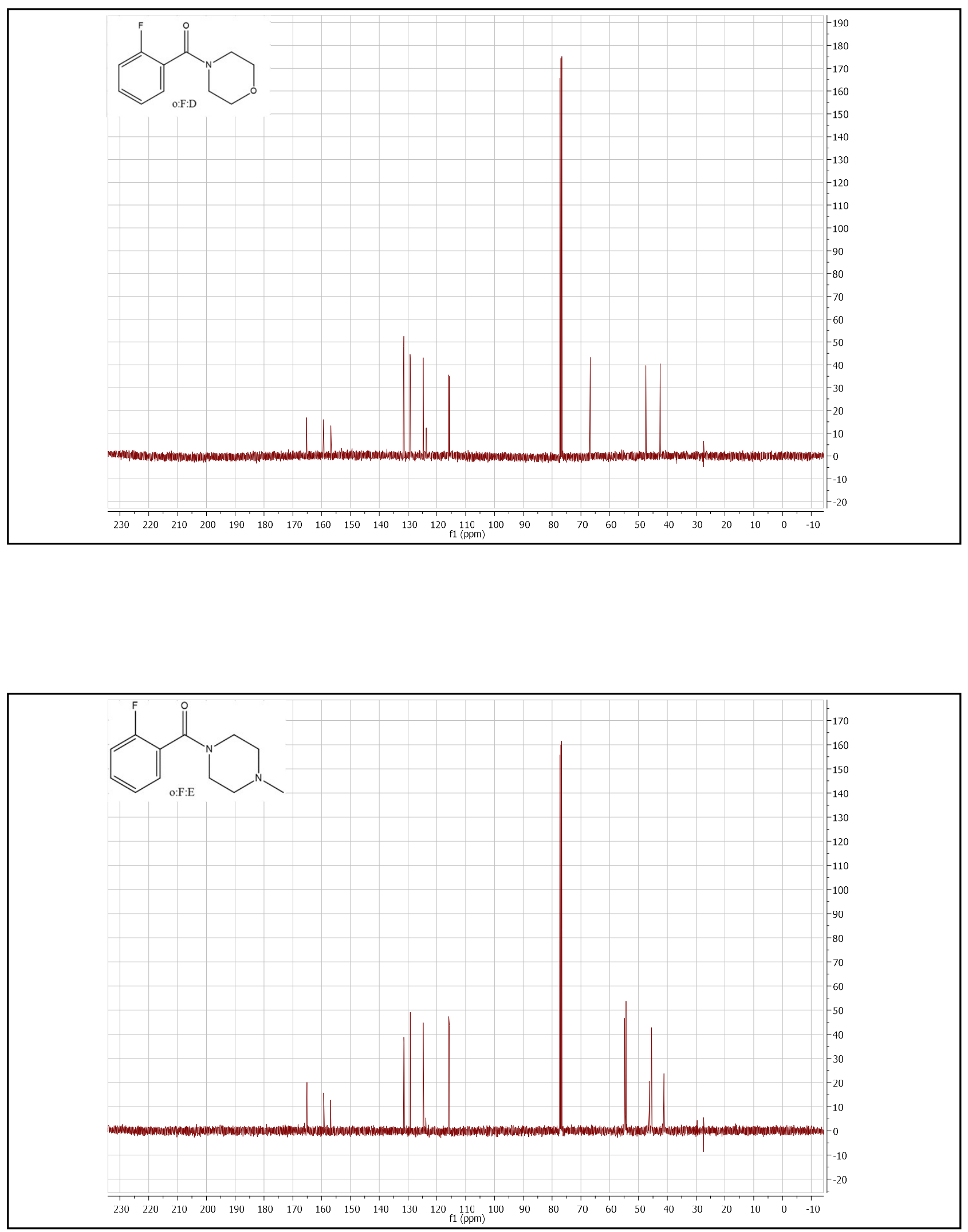

42 
Figure S2

S50
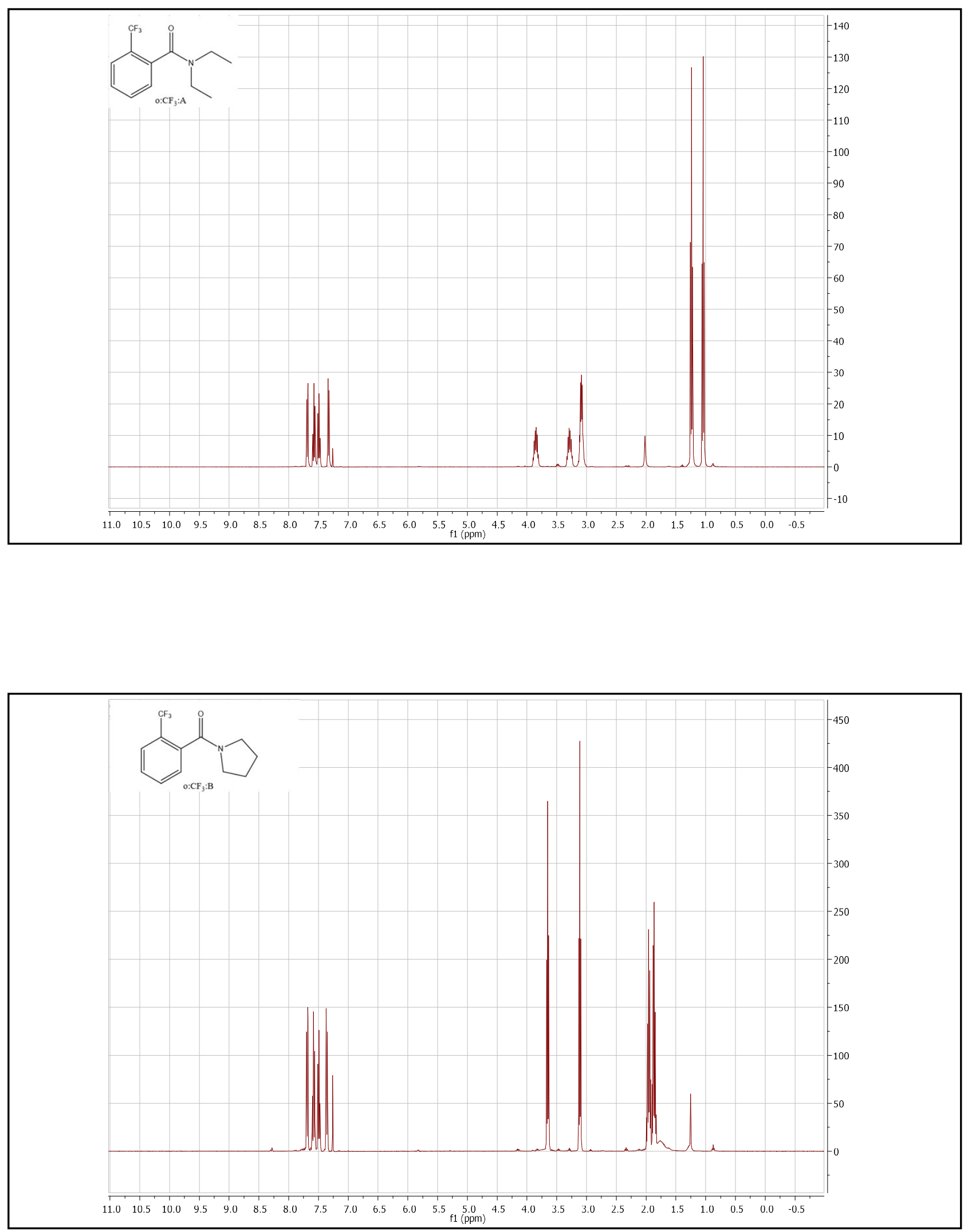

43 
Figure S2

S51
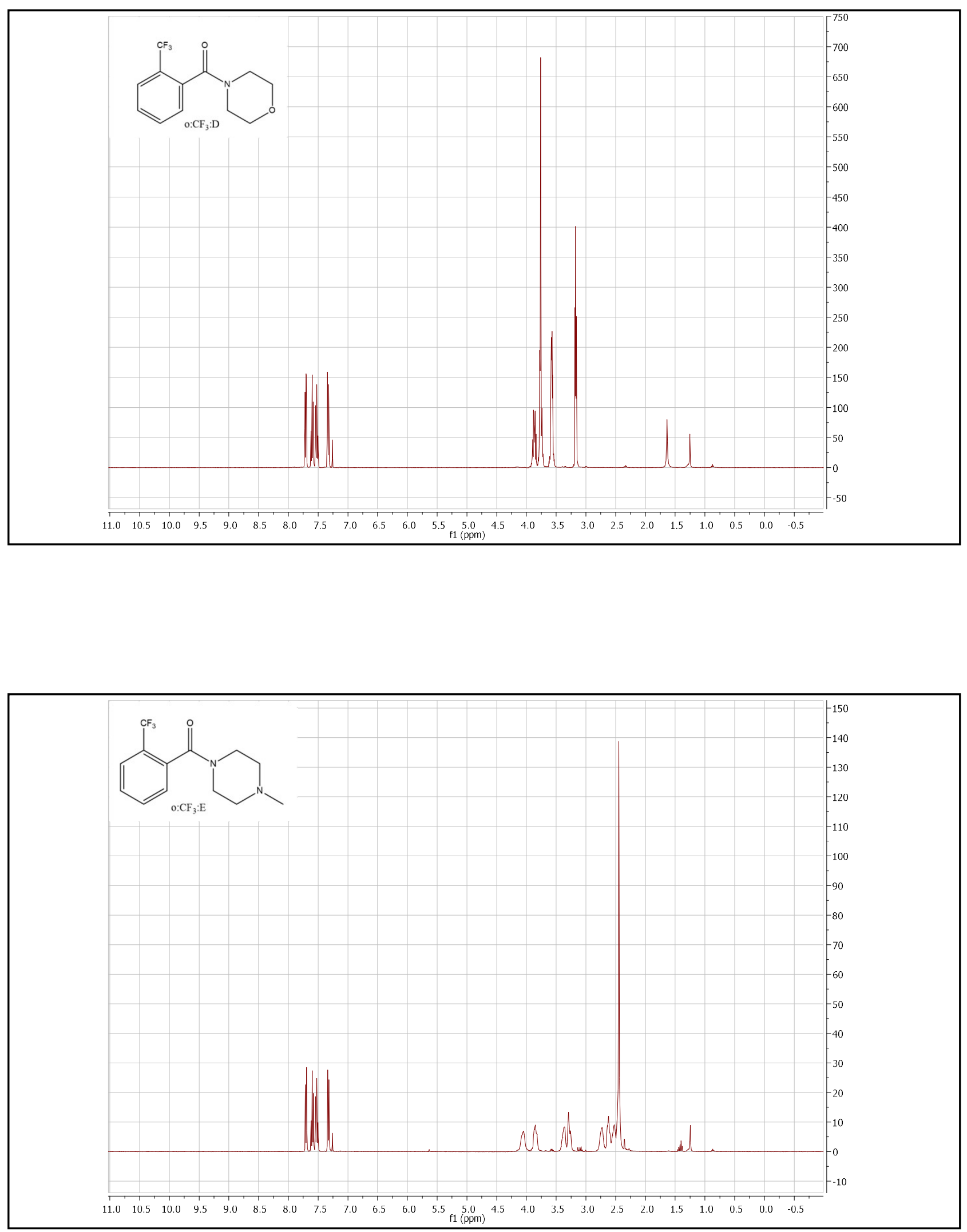

44 
Figure S2

S52
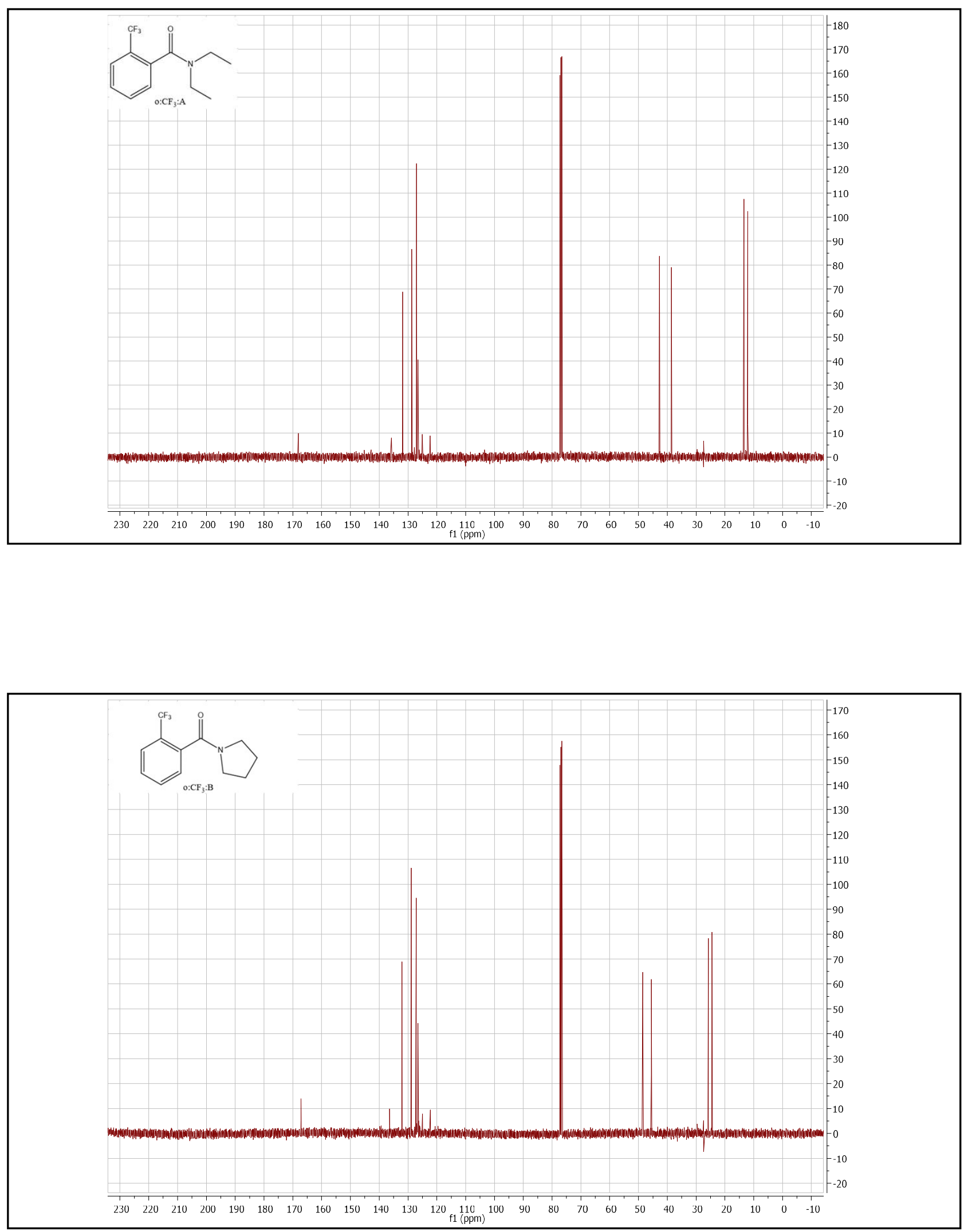

45 
Figure S2

S53
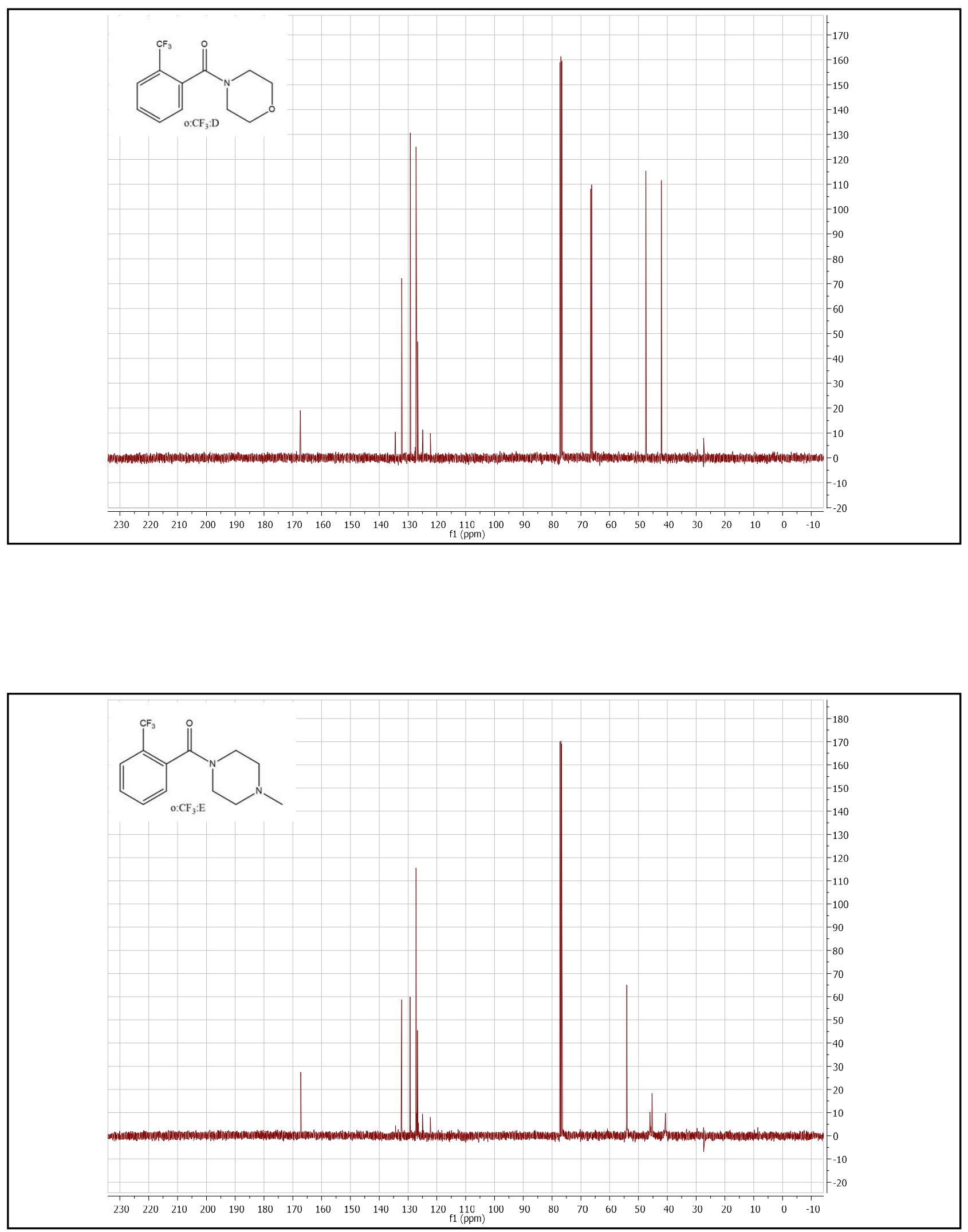

46 
Figure S2

S54
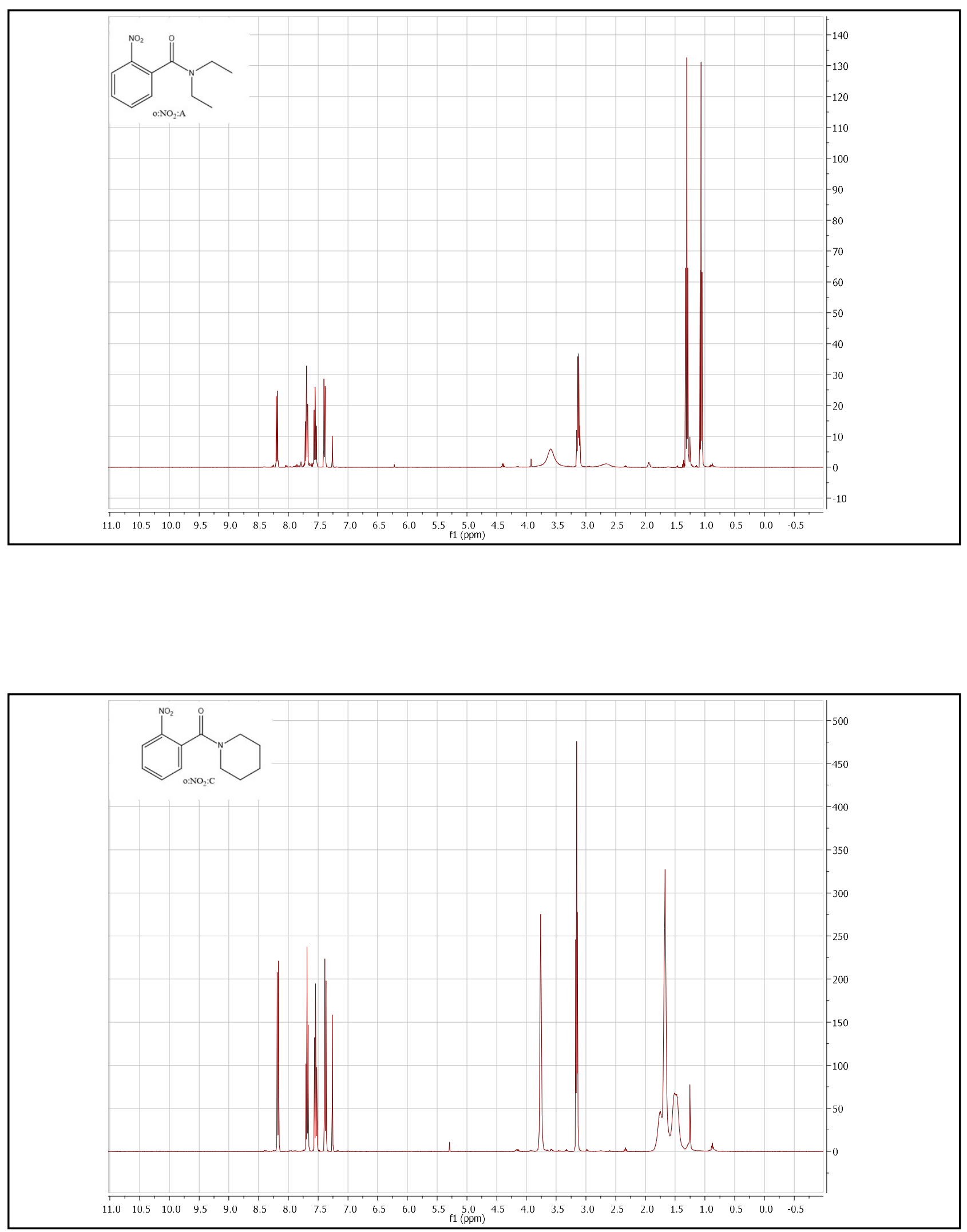

47 
Figure S2

S55
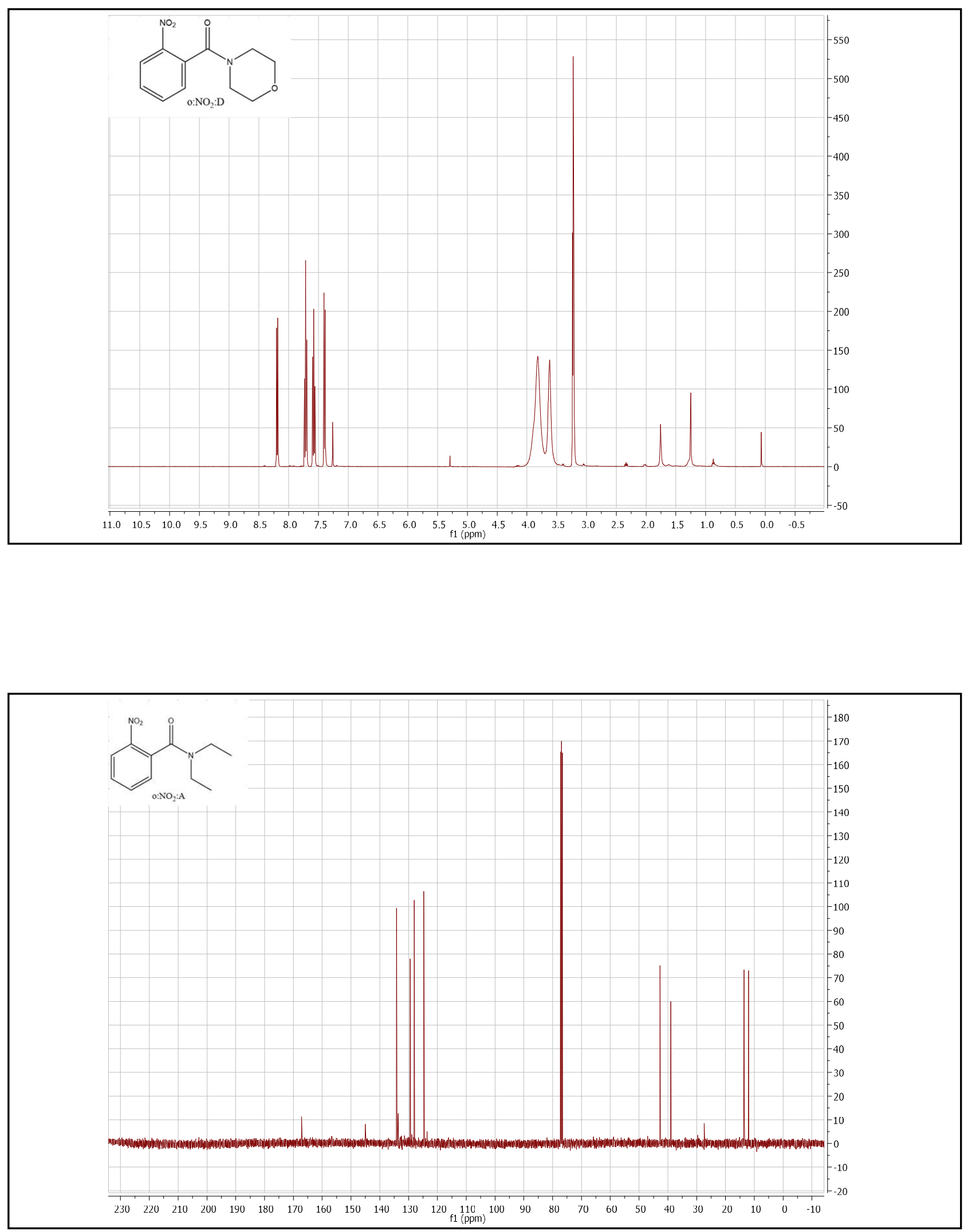

48 
Figure S2

S56
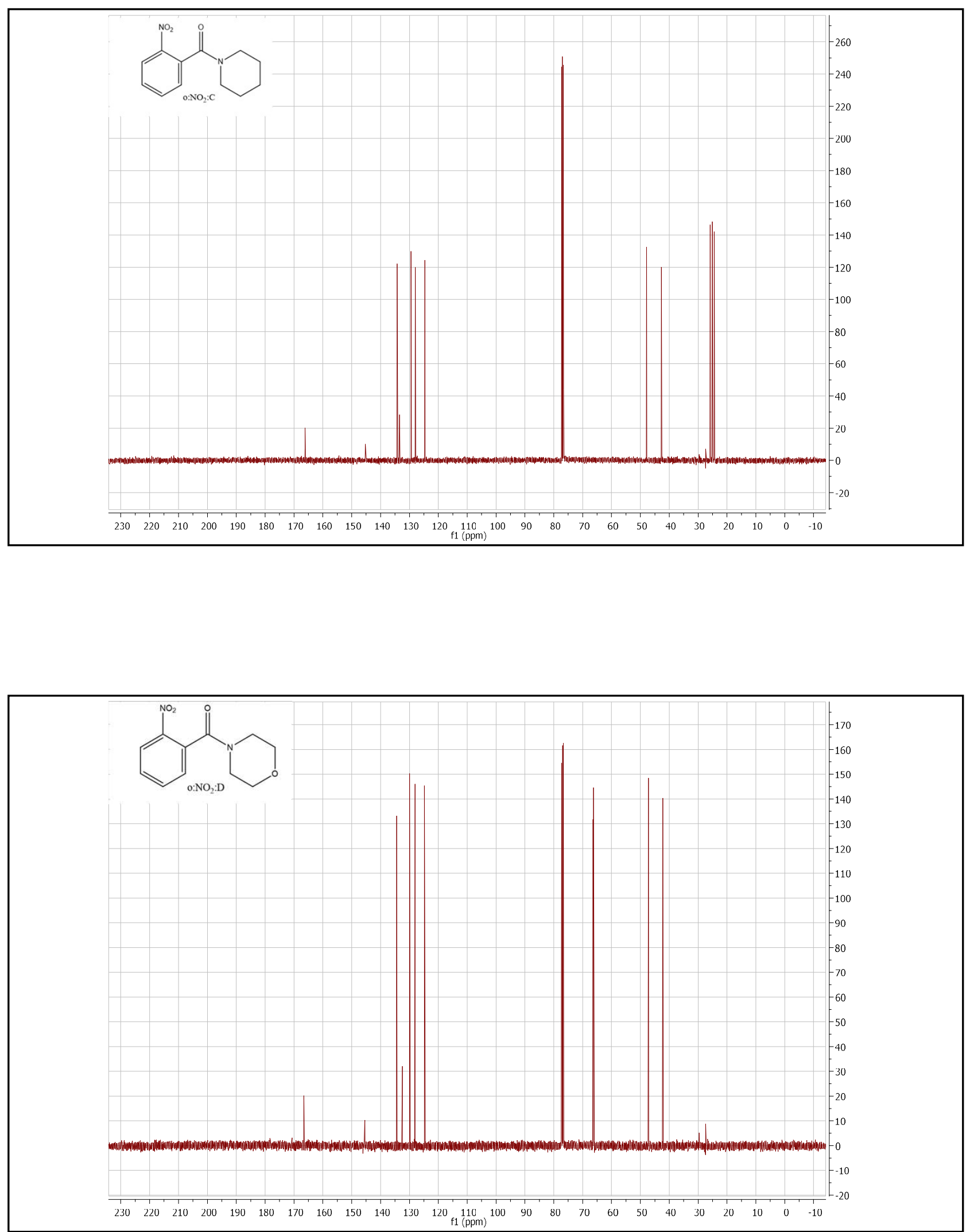

49 
Figure S2

S57
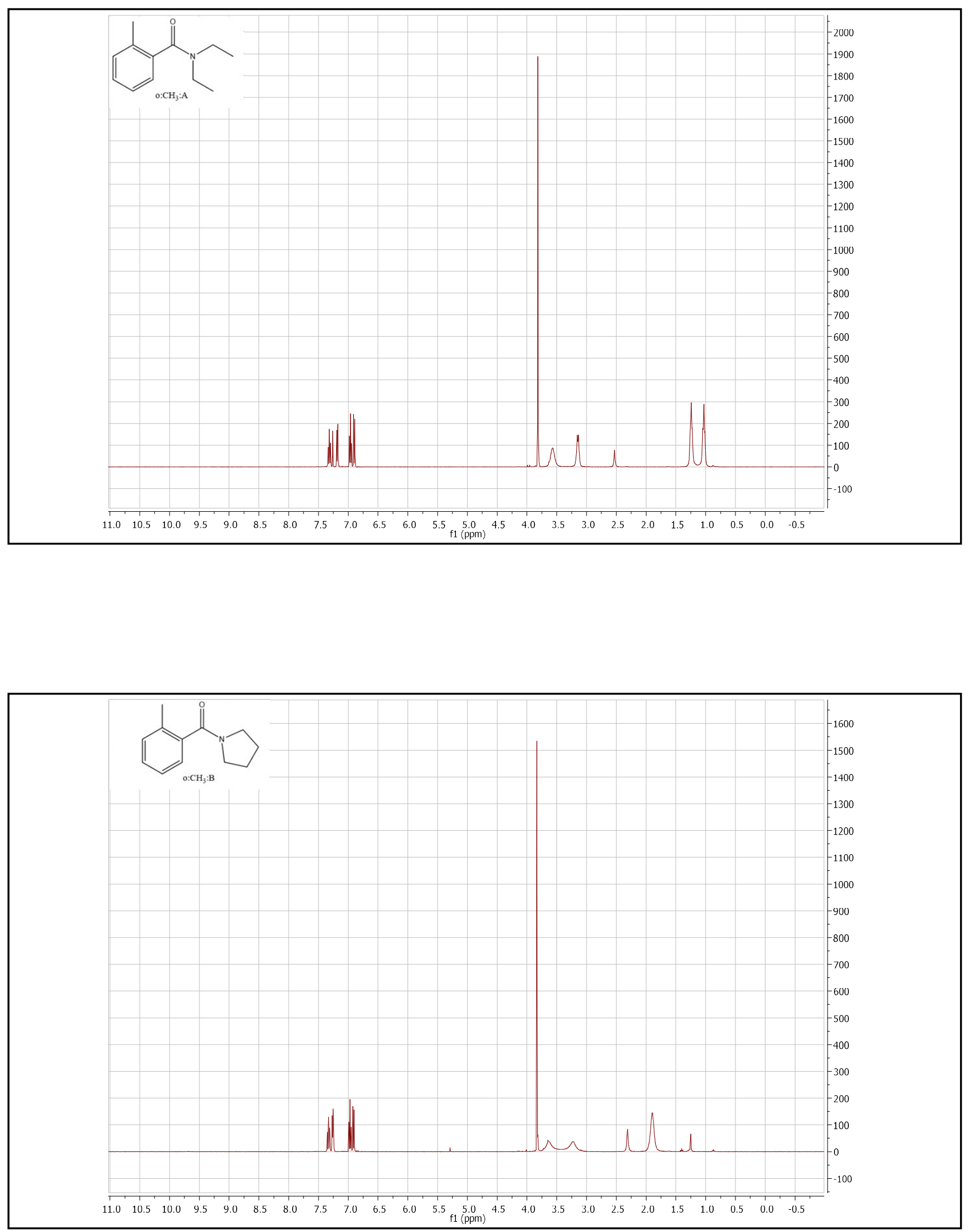

50 
Figure S2

S58
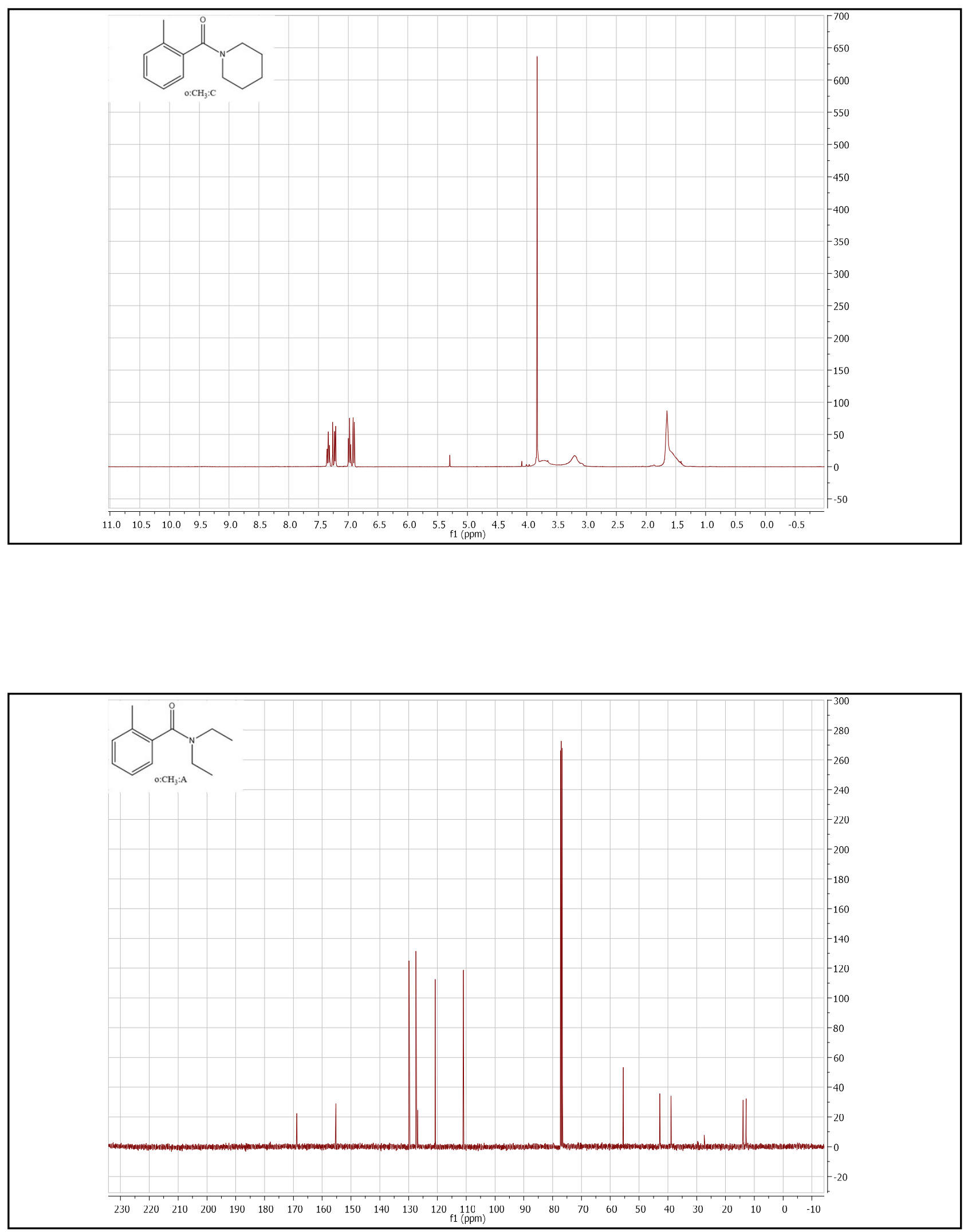

51 
Figure S2

S59
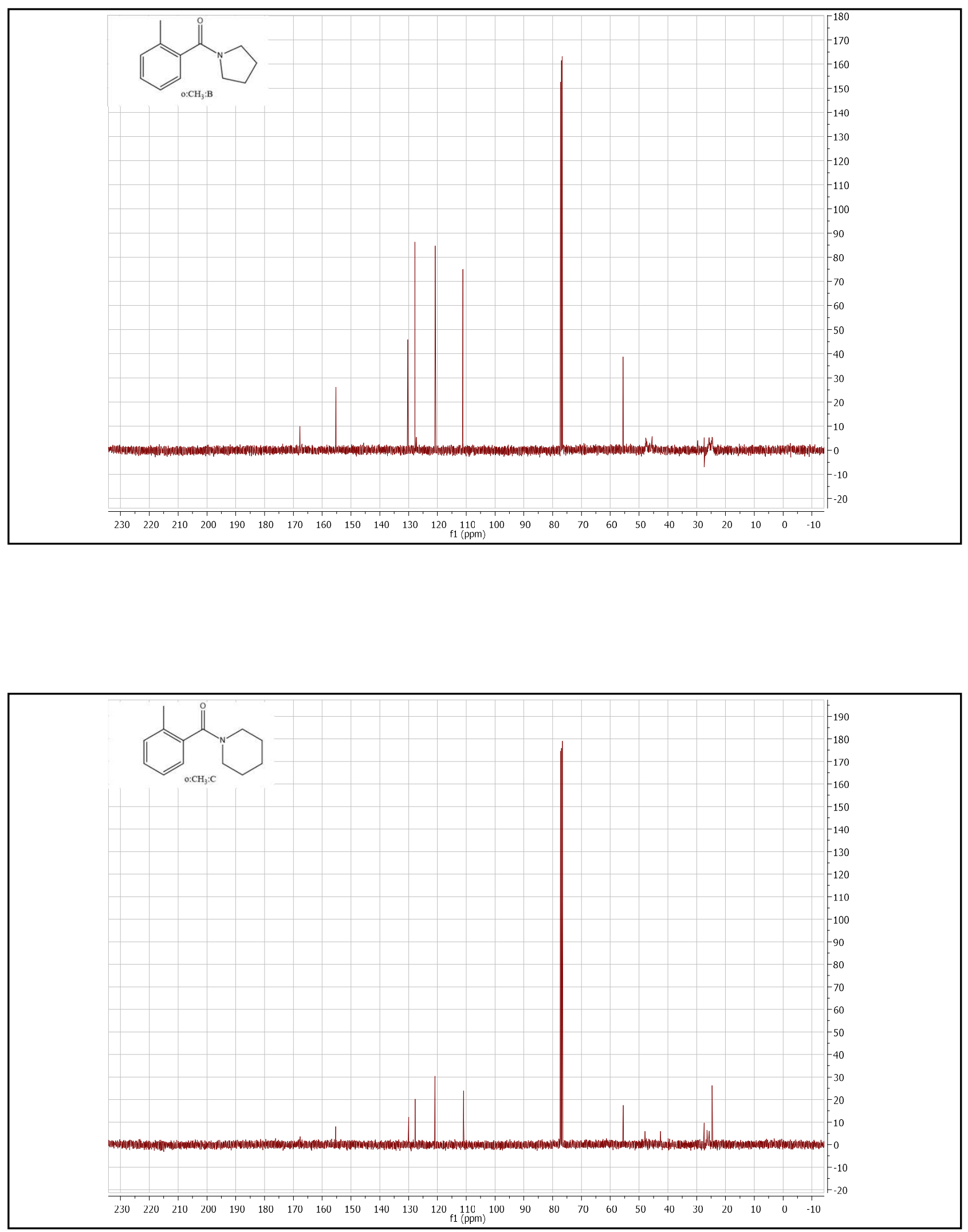

52 
Figure S2

S60
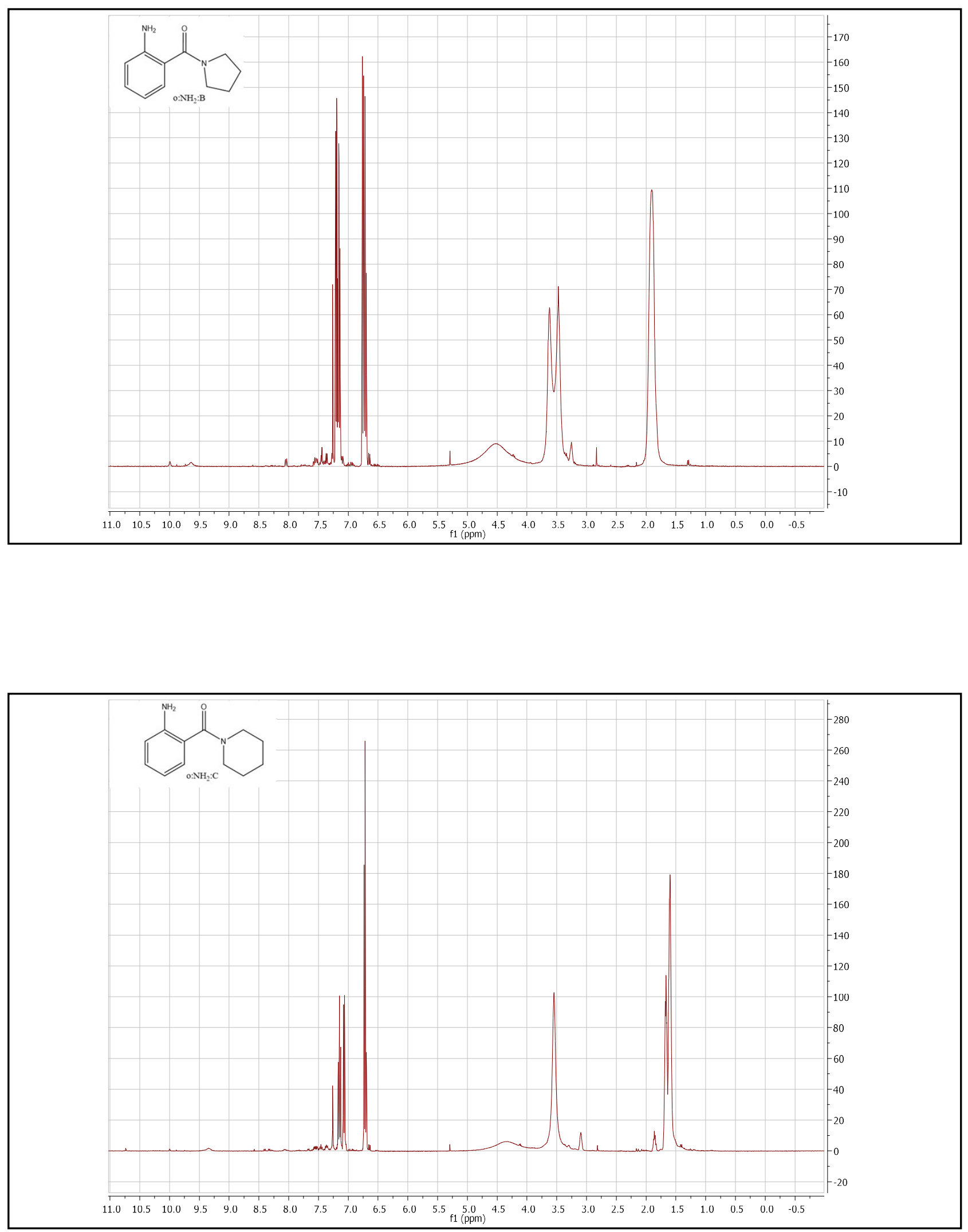

53 


$$
\frac{11}{41}
$$

